TOPICAL REVIEW

\title{
Analytic approaches to periodically driven closed quantum systems: methods and applications
}

To cite this article: Arnab Sen et al 2021 J. Phys.: Condens. Matter 33443003

View the article online for updates and enhancements.

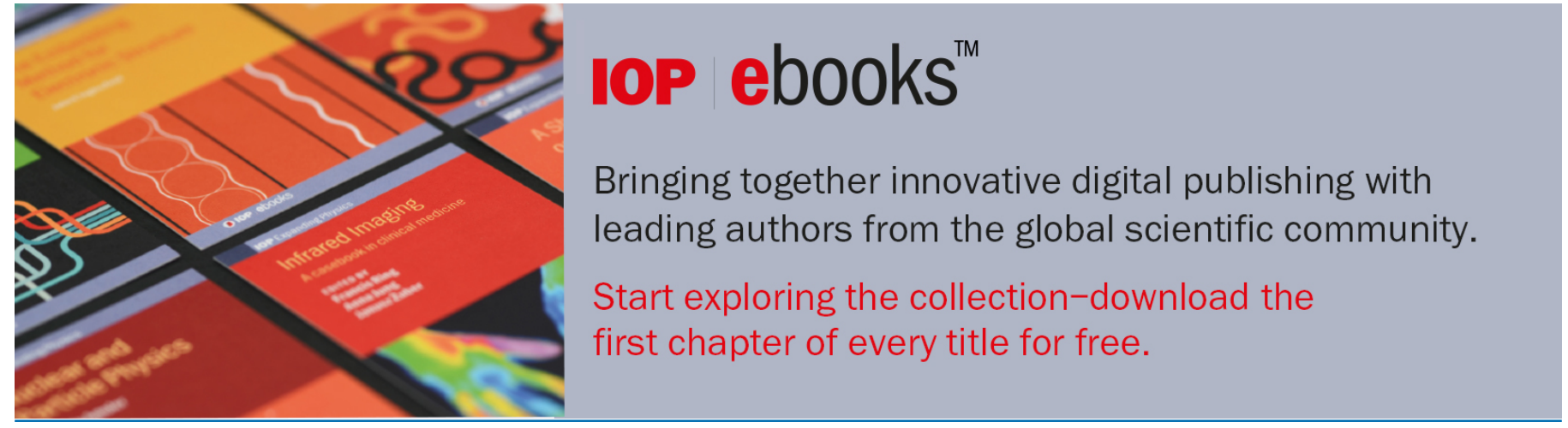

This content was downloaded from IP address 14.139 .128 .34 on 07/10/2021 at 08:30 


\title{
Analytic approaches to periodically driven closed quantum systems: methods and applications
}

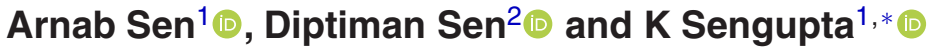 \\ ${ }^{1}$ School of Physical Sciences, Indian Association for the Cultivation of Science, 2A and 2B Raja S C \\ Mullick Road, Jadavpur 700032, India \\ 2 Center for High Energy Physics and Department of Physics, Indian Institute of Science, Bengaluru \\ 560012, India \\ E-mail: tpks@iacs.res.in
}

Received 9 February 2021, revised 19 July 2021

Accepted for publication 6 August 2021

Published 24 August 2021

\begin{abstract}
We present a brief overview of some of the analytic perturbative techniques for the computation of the Floquet Hamiltonian for a periodically driven, or Floquet, quantum many-body system. The key technical points about each of the methods discussed are presented in a pedagogical manner. They are followed by a brief account of some chosen phenomena where these methods have provided useful insights. We provide an extensive discussion of the Floquet-Magnus (FM) expansion, the adiabatic-impulse approximation, and the Floquet perturbation theory. This is followed by a relatively short discourse on the rotating wave approximation, a FM resummation technique and the Hamiltonian flow method. We also provide a discussion of some open problems which may possibly be addressed using these methods.
\end{abstract}

Keywords: many-body theory, non-equilibrium dynamics, Floquet Hamiltonian, periodically driven systems

(Some figures may appear in colour only in the online journal)

\section{Introduction}

The physics of periodically driven, or Floquet, quantum manybody systems has received tremendous attention in recent times [1-6]. This is due to the fact that such driven systems exhibit a gamut of interesting phenomena which have no analogs either in equilibrium closed quantum systems or in systems taken out of equilibrium using quench or ramp protocols [7, 8]. Moreover, in recent times ultracold atoms in optical lattices, trapped ions, superconducting qubits and magnetic

* Author to whom any correspondence should be addressed. systems have provided the much needed experimental platforms where theoretical results involving such driven systems can be tested [9-17].

In periodically driven quantum many-body systems, where the time-dependent Hamiltonian follows $H(t+n T)=H(t)$ for a fixed time period $T=2 \pi / \omega_{\mathrm{D}}$ with $\omega_{\mathrm{D}}$ being the associated drive frequency and $n$ being an arbitrary integer, the stroboscopic dynamics at times $t=n T$ is controlled by the Floquet Hamiltonian $H_{\mathrm{F}}$ [18]. The Floquet Hamiltonian is a Hermitian operator defined as the generator of the single-period timeevolution operator, or the Floquet unitary $U(T)$, which equals

$$
U(T)=\mathcal{T}\left[\mathrm{e}^{-\mathrm{i} \int_{0}^{T} \mathrm{~d} t H(t) / \hbar}\right]=\mathrm{e}^{-\mathrm{i} H_{\mathrm{F}} T / \hbar},
$$


where $\mathcal{T}$ denotes time-ordering. We note here that the timeordering makes it notoriously difficult to calculate $H_{\mathrm{F}}$ for interacting systems and one generally has to resort to various approximations.

Most of the phenomena in periodically driven closed quantum systems which have attracted recent attention follow from the properties of their corresponding Floquet Hamiltonians. For example, these Hamiltonians in periodically driven systems may have topologically non-trivial eigenstates even when the ground state of the equilibrium system is topologically trivial [19-26]. Thus the drive may generate non-trivial topology which can be characterized by specific topological invariants [22-24]. Such systems are also known to exhibit dynamical transitions that arise from a change of the properties of their Floquet Hamiltonian as a function of the drive frequency; these transitions have no analog in quantum system in equilibrium $[27,28]$. Periodic driving allows one to carry out Floquet engineering of the band structure of a system, for instance, by producing transitions between a non-topological insulator, a topological insulator and a semimetal in phosphorene [24], changing the isotropic Dirac dispersion to an anisotropic or gapped or flat dispersion in graphene [29, 30], and changing the nature of transport at the edges of a spin Hall insulator from dissipationless to dissipative [31]. Moreover, periodically driven systems may lead to the realization of a time-crystalline state of matter (a phase of matter which is disallowed in equilibrium [32]); such a state (for a discrete time crystal characterized by a $Z_{n}$ symmetry group) exhibits discrete broken time translational symmetry so that its local correlation functions develop $n T$-periodicity even when the Hamiltonian is $T$-periodic [33-35]. Furthermore, such driven systems exhibit dynamical freezing wherein the driven state of the system, after $n$ periods of the drive at specific frequencies remains arbitrarily closed to the initial state [36-39]. Also, it is well-known that quantum systems in the presence of a periodic drive may lead to dynamical localization where the drive leads to suppression of the transport of particles in the system [30, 40-42]. Finally, more recently, it was found that there is a class of periodic drives which respects the conformal symmetry of the underlying field theory; such driven conformal field theories lead to drive-induced emergent spatial structures in the energy density and correlation functions that have no analogs in standard non-relativistic systems with external drive [43-45].

Another interesting feature of periodically driven quantum systems can be understood from the perspective of the eigenstate thermalization hypothesis (ETH) $[3,46]$. It is generally expected that all non-integrable ergodic quantum many-body systems obey ETH in the thermodynamic limit. When driven periodically, such systems absorb energy from the drive and heat up to an infinite temperature steady state implying a featureless Floquet-ETH for local correlation functions [47, 48]. This has the interesting consequence of the Floquet unitary $U(T)$ (equation (1)) resembling a random matrix [49] with all its eigenstates mimicking random states as far as local quantities are concerned, thus leading to a featureless infinite temperature ensemble starting from all initial states. However, the approach of the system to such a steady state, namely, its prethermal behavior, in the presence of a periodic drive is not well-understood and is the subject of many recent works [50-53]. It is generally agreed upon that the time window $t_{*}$ for such a prethermal regime depends on the drive frequency $t_{*} \sim \mathrm{e}^{\hbar \omega_{\mathrm{D}} / J_{\text {loc }}}$, where $J_{\text {loc }}$ denotes a local energy scale, in the high drive frequency limit [50]. However, the extent of this regime and the Floquet prethermalization mechanisms beyond high frequencies in the intermediate or low drive frequency regime are yet to be fully understood. This is a particularly relevant issue since many ETH-violating phenomena in driven finite-sized systems can be expected to occur for drive frequencies in the prethermal regime in thermodynamically large systems, and such finite-sized systems may be experimentally realized using various platforms like ultracold atoms in optical lattices.

The violation of ETH in a thermodynamic many-body system may occur due to loss of ergodicity due to the presence of a large number of constants of motion as seen in integrable systems [7] or due to strong disorder as seen in the case of systems exhibiting many-body localization [54]. When such systems are periodically driven, they reach steady states which can be qualitatively different from the standard infinite temperature steady states of their ETH obeying counterparts [55-57]. Moreover, a wide range of quantum many-body systems with constrained Hilbert spaces are known to host a special class of many-body eigenstates called quantum scars in their Hilbert space [58-61]. It has been shown that the presence of such quantum scars may change the quantum dynamics of such driven systems [58, 61]; moreover, a periodic drive applied to such systems with finite size may lead to reentrant transitions between ergodic and non-ergodic behaviors as a function of the drive frequency $[62,63]$. This phenomenon, theoretically investigated for a chain of Rydberg atoms, can be shown to follow from the property of the Floquet Hamiltonian of the driven Rydberg chains which can be experimentally realized using an ultracold atom setup [13]. Such finite chains have also been shown to exhibit both dynamical freezing and novel ETH violating steady states [38].

Though these phenomena in periodically driven quantum systems follow from the structure and properties of their Floquet Hamiltonian, the Floquet Hamiltonian of a driven quantum system can, unfortunately, be computed analytically for only a handful of cases. Therefore it is natural that several approximate methods exist in the literature which try to obtain an analytic, albeit perturbative, expression for $H_{\mathrm{F}}$ (equation (1)). These analytical results can then be compared with exact numerical studies on finite-sized systems to ascertain their accuracy and range of validity. In this review, we will explore some of these methods with a pedagogical introduction to the technical details for each followed by a short description of a few chosen areas where these method have yielded useful results. Three of these methods have been widely applied to a wide range of driven systems and therefore deserve a somewhat long discourse. These are the Floquet-Magnus (FM) expansion method (section 2), the adiabatic-impulse approximation (section 3), and the Floquet perturbation theory (FPT) (section 4). In addition, we provide somewhat shorter discussions on the rotating wave approximation (RWA), a recent FM resummation technique, and the Hamiltonian flow method in 
section 5. Finally, we summarize this review and discuss a few open problems in the field in section 6.

\section{Floquet-Magnus expansion}

In this section, we will outline the calculation of $H_{\mathrm{F}}$ in the high driving frequency regime using a technique called FM expansion $[6,64,65]$ that formally results in a series of the form

$$
H_{\mathrm{F}}=\sum_{n=0}^{\infty} T^{n} \Omega_{n} .
$$

The FM expansion is the method of choice to systematically calculate new terms in the Floquet Hamiltonian, that may be otherwise difficult to generate in an equilibrium setting, and thus manipulate out-of-equilibrium phases by controlling the drive protocol.

The explicit expressions for the first three terms in equation (2) are as follows:

$$
\begin{aligned}
\Omega_{0}= & \frac{1}{T} \int_{0}^{T} \mathrm{~d} t_{1} H\left(t_{1}\right), \\
\Omega_{1}= & \frac{1}{2 \mathrm{i} \hbar T^{2}} \int_{0}^{T} \mathrm{~d} t_{1} \int_{0}^{t_{1}} \mathrm{~d} t_{2}\left[H\left(t_{1}\right), H\left(t_{2}\right)\right], \\
\Omega_{2}= & -\frac{1}{6 \hbar^{2} T^{3}} \int_{0}^{T} \mathrm{~d} t_{1} \int_{0}^{t_{1}} \mathrm{~d} t_{2} \int_{0}^{t_{2}} \mathrm{~d} t_{3} \\
& \times\left(\left[H\left(t_{1}\right),\left[H\left(t_{2}\right), H\left(t_{3}\right)\right]\right]+\left[H\left(t_{3}\right),\left[H\left(t_{2}\right), H\left(t_{1}\right)\right]\right]\right) .
\end{aligned}
$$

The general expression for $\Omega_{n}$ (equation (2)) can be written in terms of right-nested commutators of $H(t)$ as follows:

$$
\begin{aligned}
\Omega_{n}= & \frac{1}{(n+1)^{2}} \sum_{\sigma \in \mathcal{C}_{n+1}}(-1)^{d_{b}} \frac{d_{a} ! d_{b} !}{n !} \\
& \times \frac{1}{\mathrm{i}^{n} \hbar^{n} T^{n+1}} \int_{0}^{T} \mathrm{~d} t_{1} \int_{0}^{t_{1}} \mathrm{~d} t_{2} \ldots \int_{0}^{t_{n}} \mathrm{~d} t_{n+1} \\
& \times\left[H\left(t_{\sigma(1)}\right),\left[H\left(t_{\sigma(2)}\right), \ldots,\left[H\left(t_{\sigma(n)}\right), H\left(t_{\sigma(n+1)}\right)\right] \ldots\right]\right],
\end{aligned}
$$

where $\sigma \in \mathcal{C}_{n+1}$ denotes a permutation of $\{1,2, \ldots, n+1\}$ (sum is over the $(n+1)$ ! permutations of $\left.\mathcal{C}_{n+1}\right), d_{a}\left(d_{b}\right)$ is the number of ascents (descents) in the permutation $\sigma$ where $\sigma$ has an ascent (a descent) in $i$ if $\sigma(i)<\sigma(i+1)(\sigma(i)>\sigma(i+1))$, $i=1, \ldots, n$ for $\left(i_{1} i_{2} \ldots i_{n+1}\right)=(\sigma(1) \sigma(2) \ldots \sigma(n+1))$, thus giving $d_{a}+d_{b}=n$ for any permutation $\sigma$.

We will now indicate the essential steps required for the derivation of the FM expansion (for more details, we refer the reader to references $[6,65,66]$. From standard quantum mechanics, the propagator $U\left(t, t_{0}\right)$ defined by

$$
|\psi(t)\rangle=U\left(t, t_{0}\right)\left|\psi\left(t_{0}\right)\right\rangle, \quad \text { where } \quad U\left(t_{0}, t_{0}\right)=I
$$

(here $I$ is the identity matrix and $|\psi(t)\rangle$ is the many-body wave function of the system at time $t$ ), can be expressed in terms of the Dyson series as follows:

$$
U\left(t, t_{0}\right)=I+\sum_{n=1}^{\infty} P_{n}\left(t, t_{0}\right), \quad \text { where }
$$

$$
P_{n}\left(t, t_{0}\right)=\left(\frac{-\mathrm{i}}{\hbar}\right)^{n} \int_{t_{0}}^{t} \mathrm{~d} t_{1} \ldots \int_{t_{0}}^{t_{n-1}} \mathrm{~d} t_{n} H\left(t_{1}\right) \ldots H\left(t_{n}\right) .
$$

Since $U(T)=U(T, 0)$ from equation (1), we can simply put $t_{0}=0$ and $t=T$ in equation (6) to obtain the Dyson series for $U(T)$. Note that truncating the Dyson series does not result in a unitary approximation for $U(T)$. From equations (1) and (6), it follows that

$$
H_{\mathrm{F}}=\frac{\mathrm{i} \hbar}{T} \ln \left(I+\sum_{n=1}^{\infty} P_{n}\right)
$$

where we denote $P_{n}(T, 0)$ by $P_{n}$ for brevity. Using the series expansion for the logarithm in the above expression (equation (7)), expressing the lhs using equation (2) and finally, matching terms with the same powers of $H(t)$ allows one to express $\Omega_{n}$ (equation (4)) in terms of $P_{n}$ (equation (6)). In particular, for the first few terms, we get

$$
\begin{aligned}
& \Omega_{0}=\frac{\mathrm{i} \hbar}{T} P_{1}, \\
& \Omega_{1}=\frac{\mathrm{i} \hbar}{T^{2}}\left(P_{2}-\frac{1}{2} P_{1}^{2}\right), \\
& \Omega_{2}=\frac{\mathrm{i} \hbar}{T^{3}}\left(P_{3}-\frac{1}{2}\left(P_{1} P_{2}+P_{2} P_{1}\right)+\frac{1}{3} P_{1}^{3}\right) .
\end{aligned}
$$

To express the rhs of $\Omega_{1}$ and $\Omega_{2}$ (equation (8)) in terms of rightnested commutators, we introduce the following notation:

$$
\begin{aligned}
p\left(i_{1} i_{2} \ldots i_{n}\right)= & \int_{0}^{T} \mathrm{~d} t_{1} \int_{0}^{t_{1}} \mathrm{~d} t_{2} \ldots \int_{0}^{t_{n-1}} \mathrm{~d} t_{n} \\
& \times H\left(t_{i_{1}}\right) H\left(t_{i_{2}}\right) \ldots H\left(t_{i_{n}}\right) .
\end{aligned}
$$

Using Fubini's theorem,

$$
\int_{0}^{a} \mathrm{~d} y \int_{y}^{a} F(x, y) \mathrm{d} x=\int_{0}^{a} \mathrm{~d} x \int_{0}^{x} F(x, y) \mathrm{d} y,
$$

it can then be shown that

$$
\begin{aligned}
p(1) \cdot p(1) & =p(12)+p(21), \\
p(1) \cdot p(12) & =p(123)+p(213)+p(312), \\
p(12) \cdot p(1)= & p(123)+p(132)+p(231), \\
p(1) \cdot p(1) \cdot p(1)= & p(123)+p(132)+p(213), \\
& +p(231)+p(312)+p(321) .
\end{aligned}
$$

Using equation (11) in equation (8) gives equation (3). For example, $\quad p(12)-(1 / 2)(p(1) \cdot p(1))=(1 / 2)(p(12)-p(21))$ from which the expression for $\Omega_{1}$ follows straightforwardly. It should be noted that the rhs of equation (11) contains all possible permutations of time ordering that are consistent with the time ordering within the factors of the original products on the lhs. For example, in the second line of equation (11), terms such as $p(132), p(231), p(321)$ do not appear because they are inconsistent with the time ordering implied by the lhs $p(1) \cdot p(12)$ that the second index is less than the third index while the first index is arbitrary in $p\left(i_{1} i_{2} i_{3}\right)$. This structure 
generalizes to higher orders as well allowing for the derivation of $\Omega_{n}$ in terms of right-nested commutators (equation (4)).

An important case where the integrals in equation (4) may be analytically computed is for a step-like drive between Hamiltonians $H_{1}$ for duration $T_{1}$ and $H_{2}$ for duration $T_{2}$ where $T=T_{1}+T_{2}$. equation (2) then reduces to the Baker-Campbell-Hausdorff (BCH) formula where

$$
\begin{aligned}
Z & =\ln (\exp (X) \exp (Y)) \\
& =X+Y+\frac{1}{2}[X, Y]+\frac{1}{12}[X-Y,[X, Y]]+\cdots,
\end{aligned}
$$

with the identification that $X=-i H_{1} T_{1} / \hbar, Y=-i H_{2} T_{2} / \hbar$ and $Z=-i H_{\mathrm{F}} T / \hbar$. In this section, we henceforth set $\hbar=1$ for notational convenience.

We now summarize a few general points regarding the FM expansion focussing on many-body lattice models with short-ranged Hamiltonians and bounded local Hilbert spaces $[1,5,50]$. From equations (3) and (4), it is clear that while only $\Omega_{0} \neq 0$ if $\left[H(t), H\left(t^{\prime}\right)\right]=0$ for $t \neq t^{\prime}$; in the case where $\left[H(t), H\left(t^{\prime}\right)\right] \neq 0$, the FM expansion (equation (2)) is an infinite series in general. A sufficient (but not necessary) condition for this infinite series to converge is that

$$
\frac{1}{\hbar} \int_{0}^{T} \mathrm{~d} t\|H(t)\|_{2}<\pi,
$$

where $\|A\|_{2}$ denotes the spectral norm of a matrix $A$ that equals the square root of the largest eigenvalue of the matrix $A^{\dagger} A$. For short-ranged Hamiltonians, given that the energy is extensive, we expect that $(1 / \hbar) \int_{0}^{T} \mathrm{~d} t\|H(t)\|_{2} \propto N$ where $N$ denotes the number of degrees of freedom, which implies that in general, equation (13) cannot be satisfied for any finite $T$ in a thermodynamically large system. In fact, the weight of evidence suggests that the FM expansion is indeed divergent for periodically driven interacting systems [50] which eventually heat up to a featureless infinite temperature ensemble at late times due to the absence of energy conservation under driving $[47,48]$. Assuming that the Hamiltonian has at most $k$-local terms (e.g. $k$-spin interactions in a quantum spin model on a lattice), the higher-order terms in the expansion generate progressively longer-ranged terms where $\Omega_{n}$ contains at most $n k$ local terms. Thus, taking the infinite series for the FM expansion should amount to generating a $H_{\mathrm{F}}$ that resembles a random matrix [49] and hence mimics an infinite temperature ensemble locally.

However, an important simplification happens at large drive frequencies [50] which makes truncating this divergent FM expansion up to a finite order physically meaningful. When the drive frequency $\omega_{\mathrm{D}} \gg J_{\text {loc }}$ where $J_{\text {loc }}$ denotes the energy scale associated with local rearrangements of the degrees of freedom in an interacting problem (which can be deciphered from $H(t))$, there appears a large transient time $t_{*} \sim \exp \left(\omega_{\mathrm{D}} / J_{\text {loc }}\right)$ below which the system is in a prethermal Floquet state that can be well described by a truncated Floquet Hamiltonian $H_{\mathrm{F}}^{(n)}=\sum_{m=0}^{n} T^{m} \Omega_{m}$ choosing an optimum $n=n_{0}$. The heating is prevented in the prethermal regime $\left(t \lesssim t_{*}\right)$ because $H_{\mathrm{F}}^{(n)}$ appears as a conserved quantity at stroboscopic times, i.e. at times $t=n T$. Moreover, and very importantly, the dynamics of local observables can also be accurately described [50] by the unitary dynamics generated from the truncated Floquet Hamiltonian $H_{\mathrm{F}}^{(n)}$ when $t \lesssim t_{*}$. For times $t \gg t_{*}$, the system eventually flows to an infinite temperature ensemble. Physically, a many-body system requires $\mathrm{O}\left(\omega_{\mathrm{D}} / J_{\text {loc }}\right) \gg 1$ correlated local rearrangements to absorb a single quantum of energy from the drive when the drive frequency is large, hence implying a heating time that scales as $\exp \left(\omega_{\mathrm{D}} / J_{\text {loc }}\right)$.

We now give an example to show that non-trivial terms can be generated in the FM expansion even at low orders $\left(\Omega_{1}\right.$, etc in equation (3)) which may be otherwise difficult to generate in static settings. To this end, we consider a model of spinless fermions on a one-dimensional lattice where the Floquet driving is chosen in such a manner that the problem is dynamically localized without interactions. We then consider the interacting problem and use the FM expansion to calculate the first few terms of the Floquet Hamiltonian. Since the problem has no kinetic energy in the Floquet Hamiltonian by construction (due to the dynamical localization), these terms are entirely determined by the interaction energy scale and the driving period $T$, and generate density-dependent hoppings of the fermions as we show below [41].

To this end, let us consider the Hamiltonian

$$
\begin{aligned}
H & =H_{\mathrm{NI}}+H_{\mathrm{I}} \\
& =-\gamma \sum_{j=1}^{N}\left(c_{j}^{\dagger} c_{j+1}+\text { h.c. }\right)+V \sum_{j=1}^{N} n_{j} n_{j+1},
\end{aligned}
$$

where $n_{j}=c_{j}^{\dagger} c_{j}, c_{N+1}=c_{1}$, and the number of sites, $N$, is even. The Floquet structure is induced by a periodic kicking Hamiltonian of the form

$$
H_{\mathrm{K}}=\sum_{n=-\infty}^{\infty} \delta(t-n T)\left(\alpha N_{\mathrm{e}}-\beta N_{\mathrm{o}}\right),
$$

where $N_{\mathrm{e}}=\sum_{i \in \mathrm{e}} n_{i}$ and $N_{\mathrm{o}}=\sum_{i \in \mathrm{o}} n_{i}$ are the total number of fermions on the even and odd sites, respectively. Considering the non-interacting limit of $V=0$, and using the following special case of the $\mathrm{BCH}$ formula (equation (12)) when $[X, Y]=\zeta Y$,

$$
\exp (X) \exp (Y)=\exp (\exp (\zeta) Y) \exp (X)
$$

Since $\left[n_{j}, c_{j}\right]=-c_{j}$ and $\left[n_{j}, c_{j}^{\dagger}\right]=c_{j}^{\dagger}$, we obtain

$$
\begin{aligned}
U(T) & =U_{\mathrm{K}} U_{\mathrm{NI}} \\
& =\exp \left(-\mathrm{i}\left(\alpha N_{\mathrm{e}}-\beta N_{\mathrm{o}}\right)\right) \exp \left(-\mathrm{i} H_{\mathrm{NI}} T\right) \\
& =\exp \left(+\mathrm{i} H_{\mathrm{NI}} T\right) \exp \left(-\mathrm{i}\left(\alpha N_{\mathrm{e}}-\beta N_{\mathrm{o}}\right)\right)
\end{aligned}
$$

when $\alpha+\beta=\pi$. Restricting to $\alpha=\pi, \beta=0$ so that the periodic kicks (equation (15)) are applied only to the even sites implies that

$$
U(2 T)=U^{2}(T)=\exp \left(-\mathrm{i} 2 \pi N_{\mathrm{t}}\right)=I,
$$

where $N_{\mathrm{t}}$ is the total number of fermions in the system. Thus, the non-interacting system is strictly localized at intervals of $2 T$. We now turn on $H_{\mathrm{I}}$ (equation (14)) and compute $H_{\mathrm{F}}$ as an 
expansion in powers of the drive period $T$ (equation (2)). Since $H_{\mathrm{I}}$ commutes with $H_{\mathrm{K}}$, it can be seen that

$$
\begin{aligned}
U^{2}(T) & =\exp \left(-\mathrm{i} H_{\mathrm{F}} 2 T\right) \\
& =\exp \left(-\mathrm{i}\left(-H_{\mathrm{NI}}+H_{\mathrm{I}}\right) T\right) \exp \left(-\mathrm{i}\left(H_{\mathrm{NI}}+H_{\mathrm{I}}\right) T\right) .
\end{aligned}
$$

We can now use the $\mathrm{BCH}$ formula (equation (12)) to arrive at

$$
H_{\mathrm{F}}=H_{\mathrm{I}}+\frac{\mathrm{i} T}{2}\left[H_{\mathrm{NI}}, H_{\mathrm{I}}\right]-\frac{T^{2}}{6}\left[H_{\mathrm{NI}},\left[H_{\mathrm{NI}}, H_{\mathrm{I}}\right]\right]+\cdots,
$$

which finally gives

$$
\begin{aligned}
H_{\mathrm{F}}= & V \sum_{j} n_{j} n_{j+1}-\frac{\mathrm{i} \gamma T V}{2} \sum_{j}\left(c_{j+1}^{\dagger} c_{j}-c_{j}^{\dagger} c_{j+1}\right) \\
& \times\left(n_{j-1}-n_{j+2}\right)-\frac{\gamma^{2} T^{2} V}{3} \\
& \times \sum_{j}\left(\left(n_{j}-n_{j+1}\right)\left(n_{j-1}-n_{j+2}\right)\right. \\
& +\frac{1}{2}\left(c_{j-1}^{\dagger} c_{j+1}+c_{j+1}^{\dagger} c_{j-1}\right)\left(n_{j+2}+n_{j-2}-2 n_{j}\right) \\
& \left.-\left(c_{j-2}^{\dagger} c_{j-1}-c_{j-1}^{\dagger} c_{j-2}\right)\left(c_{j}^{\dagger} c_{j+1}-c_{j+1}^{\dagger} c_{j}\right)\right) .
\end{aligned}
$$

Thus, the Floquet Hamiltonian (equation (21)) contains density-dependent fermion hoppings and pairwise hoppings apart from the usual density-density interactions induced by $H_{\mathrm{I}}$ (equation (14)).

Before concluding this section, we briefly discuss another incarnation of Floquet prethermalization that allows the realization of prethermal versions of nonequilibrium phases like Floquet time crystals [33-35], but without the necessity of strong disorder [67]. Such a prethermalization occurs when the drive frequency $\omega_{\mathrm{D}}$ is greater than all but one of the local scales of the Hamiltonian. Let the time-dependent Hamiltonian $H(t)$ be of the form

$$
H(t)=H_{0}(t)+V(t)
$$

where both $H_{0}(t)$ and $V(t)$ are periodic functions with period $T$. Furthermore, $\lambda T \ll 1$ where $\lambda$ is the local energy scale of $V(t)$. Importantly, the term in the Hamiltonian with the large coupling (comparable to the drive frequency $\omega_{\mathrm{D}}$ ) needs to take a special form to avoid rapid heating. $H_{0}(t)$ has the property that it generates a trivial time evolution over $M$ time cycles, i.e.

$$
\begin{aligned}
X^{M} & =I, \quad \text { where } \\
X & =\mathcal{T} \exp \left(-\mathrm{i} \int_{0}^{T} \mathrm{~d} t H_{0}(t)\right) .
\end{aligned}
$$

Going to the interaction picture (where $V(t)$ is the 'interaction' term), we see that

$$
U(M T)=\mathcal{T} \exp \left(-\mathrm{i} \int_{0}^{M T} \mathrm{~d} t V_{\mathrm{int}}(t)\right),
$$

where $V_{\text {int }}(t)=U_{0}(t, 0)^{\dagger} V(t) U_{0}(t, 0)$ with $U_{0}$ being the propagator for $H_{0}(t)$. Since $U_{0}(M T)=X^{M}=I$, the time evolution operator $U(M T)$ is identical in the interaction and
Schrödinger pictures. Rescaling time as $t \rightarrow t / \lambda$, equation (24) then describes a system being periodically driven at a large frequency $\tilde{\omega}_{\mathrm{D}}=2 \pi /(\lambda M T)$ by a drive of local strength 1 where standard Floquet prethermalization results apply [50] for $\tilde{\omega}_{\mathrm{D}} \gg 1$ resulting in a large prethermal time $t_{*} \sim \exp \left(\tilde{\omega}_{\mathrm{D}}\right)$.

Generalizing the ideas in references $[67,68]$ showed that within the prethermal window, the Floquet unitary $U(T)$ can be well approximated by

$$
U(T) \approx \mathcal{V}^{\dagger}(X \exp (-\mathrm{i} D T)) \mathcal{V},
$$

where $\mathcal{V}$ is a time-independent local unitary rotation, and $D$ is a local Hamiltonian that has the property $[D, X]=0$. Hence the stroboscopic time evolution has an emergent symmetry $\mathcal{V} X \mathcal{V}^{\dagger}$ that commutes with $U(T)$ even though $H(t)$ has no such symmetry. Interesting prethermal phases can be stabilized when $X$ can be interpreted as a symmetry that can be spontaneously broken due to the choice of the initial state and the dimensionality of the system.

For example, to stabilize a prethermal Floquet time crystal, an Ising ferromagnet can be considered on the square lattice with a longitudinal field applied to break the Ising symmetry explicitly, and a time-dependent transverse field providing the periodic driving [67]. Thus,

$$
\begin{aligned}
H_{0}(t) & =-\sum_{i} h_{x}(t) \sigma_{i}^{x}, \\
V & =-J \sum_{\langle i j\rangle} \sigma_{i}^{z} \sigma_{j}^{z}-h_{z} \sum_{i} \sigma_{i}^{z} .
\end{aligned}
$$

The driving is then chosen to have the property

$$
\int_{0}^{T} \mathrm{~d} t h_{x}(t)=\frac{\pi}{2},
$$

which gives $X=\prod_{i} \sigma_{i}^{x}$ and $M=2$. This implies that $h_{x} \sim 1 / T$ and it is also assumed that $h_{z}, J \ll 1 / T$. Then $D=-J \sum_{\langle i j\rangle} \sigma_{i}^{z} \sigma_{j}^{z}+\cdots$ where $\ldots$ denote higher-order corrections that preserve the Ising symmetry since $[D, X]=0$. Starting with a short-ranged correlated state $|\psi(0)\rangle$ which breaks the Ising symmetry and which also has an initial energy density (with respect to the Hamiltonian $D$ ) that corresponds to a temperature $T<T_{\mathrm{c}}$ in two dimensions (where $T_{\mathrm{c}}$ denotes the critical temperature for spontaneous breaking of the Ising symmetry) ensures that $\left\langle\sigma_{i}^{z}(2 n T)\right\rangle=-\left\langle\sigma_{i}^{z}((2 n+1) T)\right\rangle \neq 0$. Here, we have implicitly assumed that the system locally 'thermalizes' with respect to the Hamiltonian $D$ starting from the initial state $|\psi(0)\rangle$ on a timescale $t_{\text {th }} \ll t_{*}$. Thus, the discrete time translation symmetry of the system is spontaneously broken which results in a prethermal Floquet time crystal that eventually melts away for times $t \gg t_{*}$. As long as the discrete time translation symmetry of the drive is unbroken, this prethermal phase is stable to any small perturbations in equation (26).

\section{Adiabatic-impulse approximation}

In this section, we discuss the adiabatic-impulse method. It is one of the few methods which can compute the Floquet 
Hamiltonian accurately in the low-frequency drive regime. In this sense, it is complementary to the FM expansion described in the previous section. A somewhat detailed account of this method has been presented in reference [4]. Here we will briefly discuss its salient features, chart out the basic computations involved, and discuss its recent application to integrable periodically driven systems. A more detailed discussion of possible approaches to Floquet systems at low drive frequencies is given in reference [69].

To this end, we first consider a two-level Hamiltonian given by

$$
H_{2}=\epsilon(t) \sigma_{z}+\Delta_{0} \sigma_{1},
$$

where $\sigma_{x, z}$ are Pauli matrices and $\Delta_{0}$ is a constant. We will consider $\epsilon(t)=\epsilon_{0} f\left(\omega_{\mathrm{D}} t\right)$ to be an arbitrary periodic function of time, characterized by a drive amplitude $\epsilon_{0}$ and a periodic time-dependent function $f\left(\omega_{\mathrm{D}} t\right)$, where $\omega_{\mathrm{D}}=2 \pi / T$ is the drive frequency, and $T$ is the time period. The method yields an accurate description of the system for $\epsilon_{0}^{2}+\Delta_{0}^{2} \gg\left(\hbar \omega_{\mathrm{D}}\right)^{2}$ and is thus suitable for capturing the low-frequency drive regime.

The central quantity that one aims to obtain using this technique is the unitary evolution operator $U(t, 0)$ which maps the initial state to the final state at time $t:|\psi(t)\rangle=U(t, 0)\left|\psi_{\text {in }}\right\rangle$. The adiabatic-impulse approximation allows a semi-analytic computation of $U(t, 0)$ for all $t$ and thus is suitable for describing the micromotion of the system. This also means that it provides us information about the phase bands, or instantaneous eigenvalues of $U$, of the system [22]. This feature and the applicability to low-frequency dynamics distinguishes this method from most other approximate analytical techniques for computing $H_{\mathrm{F}}$.

To chart out the computation of $U$ using this method, we first note that the instantaneous eigenvalue of $U(t, 0)$ can be trivially found from equation (28) and are given by

$$
E_{ \pm}(t)= \pm E(t), \quad E(t)=\sqrt{\epsilon(t)^{2}+\Delta_{0}^{2}} .
$$

The corresponding instantaneous eigenvectors are given by

$$
\begin{aligned}
\left|\psi_{-}(t)\right\rangle & =(u(t), v(t))^{\mathrm{T}}, \quad\left|\psi_{+}(t)\right\rangle=(-v(t), u(t))^{\mathrm{T}}, \\
u(t) & =\frac{\Delta_{0}}{D(t)}, \quad v(t)=(E(t)+\epsilon(t)) / D(t), \\
D(t) & =\sqrt{[E(t)+\epsilon(t)]^{2}+\Delta_{0}^{2}} .
\end{aligned}
$$

A plot of this instantaneous energy gap $\delta E(t)=2 E(t)$ is shown in figure 1 . The plot clearly indicates that the evolution time may be divided into two distinct regimes. In the first regime, shown in figure 1 as regions I and III, one has $\delta E^{2}(t) /|\hbar \mathrm{d} \delta E(t) / \mathrm{d} t| \gg 1$; thus as per standard Landau criterion, the system in these regions undergo near-adiabatic evolution. In the other regime, denoted in figure 1 as region II, $\delta E^{2}(t) /|\hbar \mathrm{d} \delta E(t) / \mathrm{d} t| \leqslant 1$ and the evolution leads to the production of excitations. This is the impulse region. The key approximation of the adiabatic-impulse technique which enables one to analytically compute $U$ is to treat the impulse region as one with an infinitesimal width around a minimum of the instantaneous energy gap. Since this approximation clearly becomes better at lower drive frequencies, the

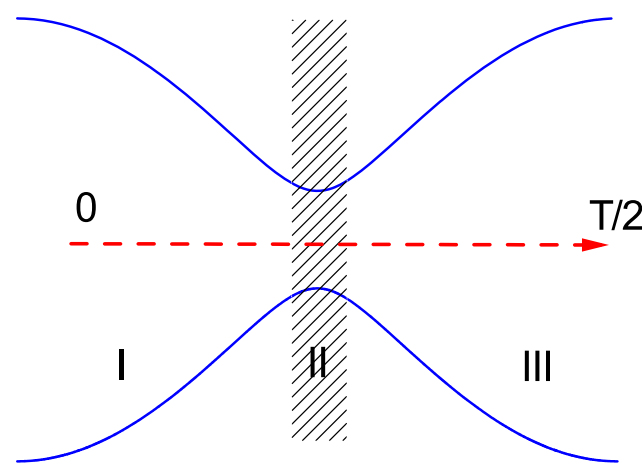

Figure 1. Schematic representation of the instantaneous energy levels of a two-level system during its evolution from $t=0$ to $t=T / 2$ with $\epsilon(t)=5 \cos t$ and $\Delta=0.5$. Regions I and III correspond to adiabatic evolution while region II (shaded area) denotes the impulse region. The width of the impulse region is taken to be close to zero in the adiabatic-impulse approximation. The red line indicates the evolution of the system for the first half-cycle from $t=0$ to $t=T / 2$. The remaining half-cycle is traversed in the opposite direction so that each region is traversed twice during evolution from $t=0$ to $t=T$.

adiabatic-impulse method naturally describes the low drivefrequency regime accurately.

To compute $U(t, 0)$ we note that the wave function of the system at any time can be expressed in the adiabatic or the moving basis as

$$
|\psi(t)\rangle=c_{1}(t)\left(\begin{array}{c}
u(t) \\
v(t)
\end{array}\right)+c_{2}(t)\left(\begin{array}{c}
-v(t) \\
u(t)
\end{array}\right),
$$

where $c_{1}(0)=1$ and $c_{2}(0)=0$. The choice of this basis makes the computation simpler, specially in the adiabatic regions I and III. We note that the basis vectors

$$
\begin{aligned}
\left|\psi_{\mathrm{ad}}^{(0)}\right\rangle & =(u(t), v(t))^{\mathrm{T}}, \\
\left|\psi_{\mathrm{ad}}^{(1)}\right\rangle & =(-v(t), u(t))^{\mathrm{T}},
\end{aligned}
$$

are related to those in the diabatic basis (given by $\left|\psi_{\text {dia }}^{(0)}\right\rangle=$ $(u(t=0), v(t=0))^{\mathrm{T}}$ and $\left.\left|\psi_{\text {dia }}^{(1)}\right\rangle=(-v(t=0), u(t=0))^{\mathrm{T}}\right)$ by the standard transformation

$$
\begin{aligned}
\left(\begin{array}{c}
\left|\psi_{\mathrm{ad}}^{(0)}\right\rangle \\
\left|\psi_{\mathrm{ad}}^{(1)}\right\rangle
\end{array}\right) & =\Lambda(t)\left(\begin{array}{c}
\left|\psi_{\mathrm{dia}}^{(0)}\right\rangle \\
\left|\psi_{\mathrm{dia}}^{(1)}\right\rangle
\end{array}\right) \\
\Lambda(t) & =\left(\begin{array}{cc}
\eta & \sqrt{1-\eta^{2}} \\
-\sqrt{1-\eta^{2}} & \eta
\end{array}\right) \\
\eta \equiv \eta(t) & =u(t) u(0)+v(t) v(0)
\end{aligned}
$$

We note that the adiabatic and the diabatic basis coincide at $t=0$ where $\eta=1$.

In region I, as discussed above, the system does not produce any excitations. Thus the dynamics leads to a kinetic phase. This can be seen most simply in the adiabatic basis where a straightforward calculation, charted out in references $[4,25,26]$ shows 


$$
\begin{aligned}
c_{1}(t) & =\exp [-\mathrm{i} \zeta(t, 0)] c_{1}(0), \\
c_{2}(t) & =\exp [\mathrm{i} \zeta(t, 0)] c_{2}(0), \\
\zeta\left(t_{1}, t_{2}\right) & =\frac{1}{\hbar} \int_{t_{2}}^{t_{1}} \mathrm{~d} t^{\prime} E\left(t^{\prime}\right) .
\end{aligned}
$$

Thus in this basis one can define $U_{I}^{\prime}(t, 0)=\exp \left[-\mathrm{i} \sigma_{z} \zeta(t, 0)\right]$ which relates $\left(c_{1}(t), c_{2}(t)\right)^{\mathrm{T}}$ to their values at $t=0$,

$$
\left(\begin{array}{l}
c_{1}(t) \\
c_{2}(t)
\end{array}\right)=U_{\mathrm{I}}^{\prime}(t, 0)\left(\begin{array}{l}
c_{1}(0) \\
c_{2}(0)
\end{array}\right) .
$$

Note that although $U_{\mathrm{I}}^{\prime}(t, 0)$ is not the true evolution operator, it acts as an useful operator which provide a handy calculational tool in the adiabatic basis. To find the true evolution operator $U_{\mathrm{I}}(t, 0)$ for all $t$ where the system is in region $\mathrm{I}$, we use equation (33) to obtain

$$
\left|\psi_{\mathrm{I}}(t)\right\rangle=\Lambda(t) U_{\mathrm{I}}^{\prime}(t, 0)\left|\psi_{\text {in }}\right\rangle=U_{\mathrm{I}}(t, 0)\left|\psi_{\text {in }}\right\rangle,
$$

where in the last line we have used the definition $\left|\psi_{\mathrm{I}}(t)\right\rangle=$ $U_{\mathrm{I}}(t, 0)\left|\psi_{\text {in }}\right\rangle$. This finally yields

$$
U_{\mathrm{I}}(t)=\left(\begin{array}{cc}
\mathrm{e}^{-\mathrm{i} \zeta(t, 0)} \eta(t) & -\mathrm{e}^{\mathrm{i} \zeta(t, 0)} \sqrt{1-\eta^{2}} \\
\mathrm{e}^{-\mathrm{i} \zeta(t, 0)} \sqrt{1-\eta^{2}} & \mathrm{e}^{\mathrm{i} \zeta(t, 0)} \eta(t)
\end{array}\right),
$$

which allows us to track the time evolution of the system in region I. A similar calculation holds for any adiabatic region.

Next, we consider region II which is reached at $t=t_{0}$ as shown in figure 1 . Here the drive leads to the production of defects. The width of this region, $\Delta t$, is approximated to be infinitesimal within the adiabatic-impulse approximation. The width of region II can be computed from the Landau criteria $\delta E^{2} \leqslant \hbar|\mathrm{d} \delta E / \mathrm{d} t|$; since $|\mathrm{d} \delta E / \mathrm{d} t| \sim \omega_{\mathrm{D}}$, it is clear that $\Delta t$ decreases with decreasing $\omega_{\mathrm{D}}$. Thus this approximation becomes better with decreasing $\omega_{\mathrm{D}}$. Typically one assumes that the width of this region is small enough so that the wave functions immediately before entering region II and immediately after leaving it are related by a transfer matrix $\mathcal{N}$

$$
\left(\begin{array}{l}
c_{1}\left(t_{0}+\Delta t\right) \\
c_{2}\left(t_{0}+\Delta t\right)
\end{array}\right)=\mathcal{N}\left(\begin{array}{l}
c_{1}\left(t_{0}-\Delta t\right) \\
c_{2}\left(t_{0}-\Delta t\right)
\end{array}\right) .
$$

To compute $\mathcal{N}$, one typically uses a linearized description of $H$. Within this approximation one writes $H(t) \simeq\left[\epsilon\left(t_{0}\right)+\right.$ $\left.\left(t-t_{0}\right) \dot{\epsilon}\left(t_{0}\right)\right] \sigma_{z}+\sigma_{x} \Delta_{0}$, where $t_{0}$ is the time at which the system reaches region II. The linearization of $H(t)$ around $t=t_{0}$ reduces the problem to that of computing the probability of the generation of defects due to Kibble-Zureck mechanism [7]. It is well-known that the probability $p$ of defect formation in this case is given by

$$
p=\exp [-2 \pi \delta], \quad \delta=\Delta_{0}^{2} /\left[2 \hbar \dot{\epsilon}_{0}\left(t_{0}\right)\right]
$$

So for the two-level system, the probability for the system to remain in its starting state after crossing the impulse region is $1-p$. Thus the diagonal elements of $\mathcal{N}$ yields $N_{11}$, $N_{22} \sim \sqrt{1-p}$ while its off-diagonal element satisfies $N_{12}, N_{21} \sim \sqrt{p}$. The detailed computation of $\mathcal{N}$ from these considerations has been charted out in references $[4,26,70$, 71] and yields

$$
\begin{aligned}
\mathcal{N} & =\left(\begin{array}{cc}
\sqrt{1-p} \mathrm{e}^{-\mathrm{i} \phi_{0}} & -\sqrt{p} \\
\sqrt{p} & \sqrt{1-p} \mathrm{e}^{\mathrm{i} \phi_{0}}
\end{array}\right), \\
\phi_{0} & =\phi_{\mathrm{st}}-\pi, \\
\phi_{\mathrm{st}} & =\frac{\pi}{4}+\delta \ln \delta+\arg \Gamma[1-\mathrm{i} \delta],
\end{aligned}
$$

where $\phi_{\text {st }}$ is the Stoke's phase and $\Gamma$ denotes the gamma function.

At the end of region II, one can write

$$
\left(\begin{array}{l}
c_{1}\left(t_{0}+\Delta t\right) \\
c_{2}\left(t_{0}+\Delta t\right)
\end{array}\right)=\mathcal{N} U_{\mathrm{I}}^{\prime}\left(t_{0}-\Delta t, 0\right)\left(\begin{array}{l}
1 \\
0
\end{array}\right) .
$$

Thus the evolution operator after the system has traversed region II is given by

$$
U_{\mathrm{II}}\left(t_{0}+\Delta t, 0\right)=\Lambda\left(t_{0}+\Delta t\right) \mathcal{N} U_{\mathrm{I}}^{\prime}\left(t_{0}-\Delta t, 0\right) .
$$

This procedure can be continued to obtain $U(t, 0)$ for all $t \leqslant$ $T$. To this end, we note that the system crosses the impulse region twice, at $t=t_{0}$ and $t=T-t_{0}$; the rest of the dynamics consists of passing through adiabatic regions. The evolution operator during any time $t$ can be written as

$$
\begin{aligned}
U(t, 0)= & \Lambda(t) U_{\mathrm{I}}^{\prime}(t, 0), \quad t \leqslant t_{0} \\
= & \Lambda(t) U_{\mathrm{I}}^{\prime}\left(t, t_{0}\right) \mathcal{N} U_{\mathrm{I}}^{\prime}\left(t_{0}, 0\right), \quad T-t_{0} \leqslant t \leqslant t_{0} \\
= & \Lambda(t) U_{\mathrm{I}}^{\prime}\left(t, T-t_{0}\right) \mathcal{N}^{\mathrm{T}} U_{\mathrm{I}}^{\prime}\left(T-t_{0}, t_{0}\right) \\
& \times \mathcal{N} U_{\mathrm{I}}^{\prime}\left(t_{0}, 0\right), \quad T \leqslant t \leqslant T_{0}-t .
\end{aligned}
$$

where $\mathcal{N}^{\mathrm{T}}$ denotes the transpose of $\mathcal{N}$. Thus this method may be used to compute $U(t, 0)$ for all $t \leqslant T$ and thus obtain information about the micromotion.

The instantaneous eigenvalues $\lambda_{ \pm}(t)=\exp [ \pm \mathrm{i} \theta(t)]$ of the evolution operator $U(t, 0)$ are called phase bands. They play an important role in charting out possible topological transitions in driven many-body systems [19, 22]. Moreover, at $t=T$, one can read off the eigenvalues of the Floquet Hamiltonian from them: $\lambda_{ \pm}(T)=\exp \left[ \pm \mathrm{i} \epsilon_{\mathrm{F}} T / \hbar\right]$. A straightforward computation, charted out in references $[4,26]$ yields

$$
\begin{aligned}
\cos \theta(t)= & \eta \cos \alpha_{1}(t), \quad t \leqslant t_{0} \\
= & \eta \sqrt{1-p} \cos \alpha_{2}(t) \\
& +\sqrt{p\left(1-\eta^{2}\right)} \cos \beta_{2}(t), \quad t_{0} \leqslant t \leqslant T-t_{0} \\
= & \eta\left[p \cos \alpha_{3}(t)+(1-p) \cos \beta_{3}(t)\right] \\
& +\sqrt{p(1-p)\left(1-\eta^{2}\right)}\left[\cos \left(\alpha_{3}-\phi_{\mathrm{st}}\right)\right. \\
& \left.-\cos \left(\beta_{3}-\phi_{\mathrm{st}}\right)\right], \quad t \geqslant T_{0}-t,
\end{aligned}
$$

where we have diagonalized $U(t, 0)$ in equation (43) to obtain these expressions, and $\alpha_{1,2,3}$ and $\beta_{2,3}$ are given by

$$
\alpha_{1}(t)=\zeta(t, 0), \quad \alpha_{2}(t)=\zeta(t, 0)+\phi_{\mathrm{st}},
$$




$$
\begin{aligned}
& \beta_{2}(t)=\zeta\left(t, t_{0}\right)-\zeta\left(t_{0}, 0\right), \quad \alpha_{3}(t)=2 \phi_{\mathrm{st}}+\zeta(t, 0), \\
& \beta_{3}(t)=\zeta\left(t_{0}, 0\right)-\zeta\left(T-t_{0}, t_{0}\right)+\zeta\left(t, T-t_{0}\right) .
\end{aligned}
$$

The computational scheme charted above brings out two aspects of the method. First, it can be directly applied to a class of integrable spin models which can be written in terms of free fermions via a Jordan-Wigner transformation. These models include the one-dimensional Ising and XY models and the twodimensional Kitaev models [72, 73]. In addition, it can also be used to describe the dynamics of Dirac quasiparticles in graphene or atop a topological insulator surface [74, 75], and Weyl fermions in 3D band systems [76]. All these systems can be represented by fermionic Hamiltonians of the form

$$
H=\sum_{\vec{k}} \psi_{\vec{k}}^{\dagger} H_{\vec{k}} \psi_{\vec{k}},
$$

where $H_{\vec{k}}$ is given by equation (28) with $\epsilon_{0}(t) \rightarrow \epsilon_{\vec{k}}(t)$ and $\Delta_{0} \rightarrow \Delta_{\vec{k}}$. The precise forms of $\epsilon_{\vec{k}}(t)$ and $\Delta_{\vec{k}}$ depend on the model and are well-known [72-76]. Second, the method provides an easy access to the micromotion in these systems; thus it allows one to address the phase bands of these models. It has been recently pointed out that the understanding of topological transitions in such driven systems requires an analysis of their phase bands $\theta(\vec{k}, t)$, and a knowledge of only their Floquet spectrum $\epsilon_{\mathrm{F}}(\vec{k})=\theta(\vec{k}, T) / T$ may not be sufficient [22]. We note in passing that this scheme can be generalized to cases where both $\epsilon$ and $\Delta$ are time dependent; the details of such generalizations have been charted out in references [26, 77].

In what follows, we will provide an example of graphene in the presence of external radiation where one can use this method to detect a topological transition at $t=T / 3$ [26]. The Hamiltonian of graphene in the presence of an external radiation is given by equation (46) with $\epsilon_{\vec{k}}(t)=-\operatorname{Re} Z_{\vec{k}}(t)$ and $\Delta_{\vec{k}}(t)=\operatorname{Im} Z_{\vec{k}}(t)$, where

$$
\begin{aligned}
Z_{\vec{k}}(t)= & -\sum_{p= \pm 1} \mathrm{e}^{\mathrm{i}\left(\cos (\omega t-p \pi / 3)+\left(k_{x}+\sqrt{3} p k_{y}\right) / 2\right)} \\
& -\mathrm{e}^{\mathrm{i}\left(\cos (\omega t)-k_{x}\right)},
\end{aligned}
$$

where $\alpha=e A_{0} / c$, and $A_{0}$ and $\omega$ are the amplitude and frequency of the circularly polarized external radiation represented by the vector potential $\vec{A}=A_{0}(\cos (\omega t), \sin (\omega t))$. It can be directly checked that at the $\Gamma$ point of the Brillouin zone $\left(\left(k_{x}, k_{y}\right)=(0,0)\right), H_{\vec{k}}$ satisfies

$$
\begin{aligned}
H_{\vec{k}}(t) & =H_{\vec{k}}(T-t) \\
H_{\vec{k}}(T / 3 \pm t) & =H_{\vec{k}}(t)=H_{\vec{k}}(2 T / 3 \pm t) \\
U(2 T / 3,0) & =[U(T / 3,0)]^{2}, \quad U(T, 0)=[U(T / 3,0)]^{3},
\end{aligned}
$$

where $U$ represents the evolution operator at the $\Gamma$ point. This shows that a phase band crossing leading to a change of topology of the driven system at $t=T / 3$ (which amounts to $U(T / 3,0)= \pm I)$ necessarily shows analogous crossing at $t=T$; however, the reverse is not true.

The verification of such crossings at $t=T / 3$ and $2 T / 3$ has been carried out in detail in reference [26]. A somewhat lengthy calculation yields an analytical expression for the phase bands within the adiabatic-impulse approximation. In terms of the probability $p_{\Gamma}$ for the formation of excitations formation probability and the associated Stuckelberg phase $\Phi_{\Gamma}$, one finds that the expression for the phase band $\phi_{\Gamma}$ at the $\Gamma$ point is [26]

$$
\begin{aligned}
\cos (\phi(T / 3)) & =p_{\Gamma}+\left(1-p_{\Gamma}\right) \cos \left(2 \Lambda_{\Gamma}\right), \\
\Lambda_{\Gamma} & =\Phi_{\Gamma}+2 \int_{0}^{T / 6} \mathrm{~d} t \sqrt{\epsilon_{\vec{k}=0}^{2}(t)+\Delta_{\vec{k}=0}^{2}(t)} .
\end{aligned}
$$

It was shown that the band crossings that lead to a change in topology of the state of the driven system at $t=T / 3$ requires $\cos \left[\phi_{\Gamma}(T / 3)\right]=+(-) 1$ for crossings through the zone center (edge). The crossings through the zone center thus requires $\Lambda_{\Gamma}=m \pi$ for $m \in Z$. The crossing through the zone edge, in contrast, necessitates $\cos \left[2 \Lambda_{\Gamma}\right]=-\left(1+p_{\Gamma}\right) /\left(1-p_{\Gamma}\right)$; this is clearly untenable for real $\Lambda$ and hence the adiabatic-impulse approximation predicts that all such band crossings at $t=$ $T / 3$ should occur through the zone center. This fact has been numerically verified in reference [26]. A similar analysis has been carried out for other values of $T$ and at other points in the graphene Brillouin zone. In all cases, the prediction of the adiabatic-impulse method provides a near-exact match with exact numerics as long as the drive frequency is small compared to the nearest-neighbor hopping amplitude of the electrons in graphene; in addition it provides analytical conditions for phase band crossings which help in obtaining a semi-analytic understanding of the phase diagram of periodically driven graphene [26]. Moreover, such an analysis can be easily extended to a wide class of driven spin and fermionic systems which host Dirac-like quasiparticles. It thus provides a complete picture of the low-frequency behavior of a wide range of integrable models.

\section{Floquet perturbation theory}

In this section, we discuss a perturbative method to find the Floquet Hamiltonian $H_{\mathrm{F}}$ or periodically driven manybody Hamiltonians of the form $H(t)=H_{0}(t)+g V(t)$, where $H_{0}(t)(V(t))=H_{0}(t+T)(V(t+T))$ (note that $H_{0}(t)$ or $V(t)$ may be time-independent), $g \ll 1$, and crucially, $H_{0}(t)$ consists of mutually commuting terms. We call this FPT whereby $H_{\mathrm{F}}$ is obtained as a power series in $g[39,62,78]$. This method is particularly suited to address the nature of the Floquet Hamiltonian at intermediate and low drive frequencies, unlike the high-frequency FM expansion.

As the first example, suppose that the Hamiltonian $H$ can be written as a sum of two parts, $H(t)$ which varies periodically in time with a period $T=2 \pi / \omega_{\mathrm{D}}$, and a perturbation $V$ which is time-independent. Thus $H(t)=H_{0}(t)+V$. Since $H_{0}(t)$ commutes with itself at different times, we can work in the basis of eigenstates of $H_{0}(t)$ which are time-independent and orthonormal. We denote these as $|n\rangle$, so that $H_{0}(t)|n\rangle=E_{n}(t)|n\rangle$, and $\langle m \mid n\rangle=\delta_{m n}$.

We now find solutions of the time-dependent Schrödinger equation

$$
\mathrm{i} \hbar \frac{\partial \psi_{n}}{\partial t}=H(t) \psi_{n}(t)
$$


which satisfy the Floquet eigenstate condition

$$
\psi_{n}(T)=\mathrm{e}^{\mathrm{i} \theta_{n}} \psi_{n}(0)
$$

where $\mathrm{e}^{\mathrm{i} \theta_{n}}$ is the Floquet eigenvalue.

For $V=0$, we have

$$
\psi_{n}(t)=\mathrm{e}^{-(\mathrm{i} / \hbar) \int_{0}^{t} \mathrm{~d} t^{\prime} E_{n}\left(t^{\prime}\right)}|n\rangle,
$$

so that the eigenvalue of the Floquet unitary

$$
U=\mathcal{T} \mathrm{e}^{-(\mathrm{i} / \hbar) \int_{0}^{T} \mathrm{~d} t H(t)}
$$

is given by

$$
\mathrm{e}^{\mathrm{i} \theta_{n}}=\mathrm{e}^{-(\mathrm{i} / \hbar) \int_{0}^{T} \mathrm{~d} t E_{n}(t)} .
$$

For $V$ non-zero but small, we will develop an FPT to first order in $V$. We first consider non-degenerate perturbation theory; the meaning of non-degenerate will become clear below. We assume that the $n$th eigenstate can be written as

$$
\psi_{n}(t)=\sum_{m} c_{m}(t) \mathrm{e}^{-(\mathrm{i} / \hbar) \int_{0}^{t} \mathrm{~d} t^{\prime} E_{m}\left(t^{\prime}\right)}|m\rangle,
$$

where $c_{n}(t)=1+$ terms of order $V$ for all $t$, while $c_{m}(t)$ is of order $V$ for all $m \neq n$ and all $t$. Equation (50) then implies

$$
\begin{aligned}
\mathrm{i} \hbar \sum_{m} \dot{c}_{m}(t) \mathrm{e}^{-(\mathrm{i} / \hbar) \int_{0}^{t} \mathrm{~d} t^{\prime} E_{m}\left(t^{\prime}\right)}|m\rangle \\
\quad=V \sum_{m} c_{m}(t) \mathrm{e}^{-(\mathrm{i} / \hbar) \int_{0}^{t} \mathrm{~d} t^{\prime} E_{m}\left(t^{\prime}\right)}|m\rangle,
\end{aligned}
$$

where $\dot{c}_{m}$ denotes $\mathrm{d} c_{m} / \mathrm{d} t$. Taking the inner product of equation (56) with $\langle n|$, we find, to first order in $V$, that $\mathrm{i} \hbar \dot{c}_{n}=$ $\langle n|V| n\rangle$. Choosing $c_{n}(0)=1$, we then have

$$
c_{n}(t)=\mathrm{e}^{-(\mathrm{i} / \hbar)\langle n|V| n\rangle t} .
$$

This gives

$$
\begin{aligned}
\psi_{n}(t)= & \mathrm{e}^{-(\mathrm{i} / \hbar)\left(\langle n|V| n\rangle t+\int_{0}^{t} \mathrm{~d} t^{\prime} E_{n}\left(t^{\prime}\right)\right)}|n\rangle \\
& +\sum_{m \neq n} c_{m}(t) \mathrm{e}^{-(\mathrm{i} / \hbar) \int_{0}^{t} \mathrm{~d} t^{\prime} E_{m}\left(t^{\prime}\right)}|m\rangle .
\end{aligned}
$$

Next, taking the inner product of equation (56) with $\langle m|$, where $m \neq n$, we find, to first order in $V$, that

$$
\dot{c}_{m}=-\frac{\mathrm{i}}{\hbar}\langle m|V| n\rangle \mathrm{e}^{(\mathrm{i} / \hbar) \int_{0}^{t} \mathrm{~d} t^{\prime}\left[E_{m}\left(t^{\prime}\right)-E_{n}\left(t^{\prime}\right)\right]}
$$

(We have ignored a factor of $\mathrm{e}^{(\mathrm{i} / \hbar)\langle n|V| n\rangle t}$ on the right-hand side of equation (59) since we are only interested in terms of first order in $V$.) Integrating equation (59) gives

$$
c_{m}(T)=c_{m}(0)-\frac{\mathrm{i}}{\hbar}\langle m|V| n\rangle \int_{0}^{T} \mathrm{~d} t \mathrm{e}^{\mathrm{i} \int_{0}^{t} \mathrm{~d} t^{\prime}\left[E_{m}\left(t^{\prime}\right)-E_{n}\left(t^{\prime}\right)\right]} .
$$

Since we know that equation (58) satisfies

$$
\psi_{n}(T)=\mathrm{e}^{-(\mathrm{i} / \hbar)\left(\langle m|V| n\rangle T+\int_{0}^{T} \mathrm{~d} t E_{n}(t)\right)} \psi_{n}(0),
$$

we must have, to first order in $V$,

$$
c_{m}(T)=\mathrm{e}^{(\mathrm{i} / \hbar) \int_{0}^{T} \mathrm{~d} t\left[E_{m}(t)-E_{n}(t)\right]} c_{m}(0)
$$

for all $m \neq n$. This, along with equation (60), means that we must choose

$$
c_{m}(0)=-\frac{\mathrm{i}}{\hbar}\langle m|V| n\rangle \frac{\int_{0}^{T} \mathrm{~d} t \mathrm{e}^{(\mathrm{i} / \hbar) \int_{0}^{t} \mathrm{~d} t^{\prime}\left[E_{m}\left(t^{\prime}\right)-E_{n}\left(t^{\prime}\right)\right]}}{\mathrm{e}^{(\mathrm{i} / \hbar) \int_{0}^{T} \mathrm{~d} t\left[E_{m}(t)-E_{n}(t)\right]}-1} .
$$

We see that $c_{m}(t)$ is indeed of order $V$ provided that the denominator on the right-hand side of equation (63) does not vanish; we call this case non-degenerate. If

$$
\mathrm{e}^{(\mathrm{i} / \hbar) \int_{0}^{T} \mathrm{~d} t\left[E_{m}(t)-E_{n}(t)\right]}=1,
$$

we have a resonance between states $|m\rangle$ and $|n\rangle$, and the above analysis breaks down. We then have to develop a degenerate FPT as discussed below.

If there are several states which are connected to $|n\rangle$ by the perturbation $V$, equation (63) describes the amplitude to go to each of them from $|n\rangle$. Up to order $V^{2}$, the total probability of excitation away from $|n\rangle$ is given by $\sum_{m \neq n}\left|c_{m}(0)\right|^{2}$. If $c_{m}(0)$ turns out to be zero for all $m \neq n$ (this can happen if either the matrix element $\langle m|V| n\rangle=0$ or the numerator of the expression in equation (63) vanishes), the Floquet eigenstate remains equal to $\psi_{n}$ up to first order in $V$. This is an example of dynamical freezing.

Next we consider degenerate perturbation theory. Suppose that there are $p$ states $|m\rangle(m=1,2, \ldots, p)$ which have energies $E_{m}$ and satisfy equation (64) for every pair of states $(m, n)$ lying in the range 1 to $p$. Ignoring all the other states for the moment, we assume that a solution of the Schrödinger equation is given by

$$
\psi_{n}(t)=\sum_{m=1}^{p} c_{m}(t) \mathrm{e}^{-(\mathrm{i} / \hbar) \int_{0}^{t} \mathrm{~d} t^{\prime} E_{m}\left(t^{\prime}\right)}|m\rangle,
$$

where we now allow all the $c_{m}(t)$ 's to be of order 1 . To first order in $V$, we can then replace $c_{m}(t)$ by the time-independent constants $c_{m}(0)$ on the right-hand side of equation (56). Upon integrating from $t=0$ to $T$, we obtain

$$
\begin{aligned}
c_{m}(T)= & c_{m}(0)-\frac{\mathrm{i}}{\hbar} \sum_{n=1}^{p}\langle m|V| n\rangle \\
& \times \int_{0}^{T} \mathrm{~d} t \mathrm{e}^{(\mathrm{i} / \hbar) \int_{0}^{t} \mathrm{~d} t^{\prime}\left[E_{m}\left(t^{\prime}\right)-E_{n}\left(t^{\prime}\right)\right]} c_{n}(0) .
\end{aligned}
$$

This can be written as a matrix equation

$$
c(T)=\left[I-\frac{\mathrm{i} H^{\prime} T}{\hbar}\right] c(0),
$$

where $c(t)$ denotes the column $\left(c_{1}(t), c_{2}(t), \ldots, c_{p}(t)\right)^{\mathrm{T}}$ (where the superscript $\mathrm{T}$ denotes transpose), $I$ is the $p$-dimensional identity matrix, and $H^{\prime}$ is a $p$-dimensional Hermitian matrix with matrix elements

$$
\left(H^{\prime}\right)_{m n}=\frac{\langle m|V| n\rangle}{T} \int_{0}^{T} \mathrm{~d} t \mathrm{e}^{(\mathrm{i} / \hbar) \int_{0}^{t} \mathrm{~d} t^{\prime}\left[E_{m}\left(t^{\prime}\right)-E_{n}\left(t^{\prime}\right)\right]} .
$$


Let the eigenvalues of $H^{\prime}$ be $\epsilon_{n}(n=1,2, \ldots, p)$. To first order in $V, I-\mathrm{i} H^{\prime} T / \hbar$ is a unitary matrix and therefore has eigenvalues of the form $\mathrm{e}^{-\mathrm{i} \epsilon_{n} T / \hbar}$; the corresponding eigenstates satisfy

$$
c(T)=\mathrm{e}^{-\mathrm{i} \epsilon_{n} T / \hbar} c(0) .
$$

Next, we want the wave function in equation (65) to satisfy equation (51). This implies that the Floquet eigenvalues are related to the eigenvalues of $H^{\prime}$ as

$$
\mathrm{e}^{\mathrm{i} \theta_{n}}=\mathrm{e}^{-(\mathrm{i} / \hbar)\left(\epsilon_{n} T+\int_{0}^{T} \mathrm{~d} t E_{n}(t)\right)},
$$

where we have used the resonance condition that $\mathrm{e}^{-(\mathrm{i} / \hbar) \int_{0}^{T} \mathrm{~d} t E_{n}(t)}$ has the same value for all $n=1,2, \ldots, p$.

Given a Floquet unitary $U(T)$, we can define a Floquet Hamiltonian $H_{\mathrm{F}}$ using equation (1). Comparing this with equations (67) and (68), we see that the matrix elements of $H_{\mathrm{F}}$ are

$$
\begin{aligned}
\left(H_{\mathrm{F}}\right)_{m n}= & \frac{\langle m|V| n\rangle}{T} \int_{0}^{T} \mathrm{~d} t \mathrm{e}^{(\mathrm{i} / \hbar)} \int_{0}^{t} \mathrm{~d} t^{\prime}\left[E_{m}\left(t^{\prime}\right)-E_{n}\left(t^{\prime}\right)\right] \\
& +\left(\frac{1}{T} \int_{0}^{T} \mathrm{~d} t E_{n}(t)\right) \delta_{m n}
\end{aligned}
$$

where we have assumed that $\int_{0}^{T} \mathrm{~d} t E_{n}(t)$ has the same value for all $n$. (This is a special case of equation (64). More generally, equation (64) allows the values of $\int_{0}^{T} \mathrm{~d} t E_{n}(t)$ to differ from each other by non-zero integer multiples of $2 \pi \hbar$, but we will not consider that possibility here.)

Unlike the FM expansion [1, 79], FPT does not assume the drive frequency $\omega_{\mathrm{D}}$ to be large compared to the other parameters of the system. It only assumes the amplitude of the driving to be large. This will become clear in the examples discussed below where we will see that the Floquet Hamiltonian is effectively an expansion in the inverse of the driving amplitude.

As the first application of the above formalism, we consider a simple model with a single spin-1/2 which is governed by a Hamiltonian $H(t)=H_{0}(t)+V$, where [80]

$$
\begin{gathered}
H_{0}=\lambda \cos (\omega t) \sigma^{x}, \\
V=g_{1} \sigma^{x}+g_{2} \sigma^{y},
\end{gathered}
$$

and we will assume that $\lambda \gg g_{1}, g_{2}$. The unperturbed problem given by $\mathrm{i} \hbar \partial \psi / \partial t=H_{0} \psi$ has solutions

$$
\begin{aligned}
\psi_{1}(t) & =\mathrm{e}^{-\mathrm{i}(\lambda / \hbar \omega) \sin (\omega t)}|1\rangle, \\
\psi_{2}(t) & =\mathrm{e}^{\mathrm{i}(\lambda / \hbar \omega) \sin (\omega t)}|2\rangle, \\
\text { where } \quad|1\rangle & =\frac{1}{\sqrt{2}}\left(\begin{array}{l}
1 \\
1
\end{array}\right) \quad \text { and }|2\rangle=\frac{1}{\sqrt{2}}\left(\begin{array}{c}
1 \\
-1
\end{array}\right),
\end{aligned}
$$

and the corresponding eigenvalues of $H_{0}(t)$ are $E_{1}(t)=$ $\lambda \cos (\omega t)$ and $E_{2}(t)=-\lambda \cos (\omega t)$ respectively. Since these satisfy equation (64) we have to use degenerate perturbation theory. Following equations (65)-(71), and using the identity [81]

$$
\int_{0}^{T} \mathrm{~d} t \mathrm{e}^{(\mathrm{i} / \hbar) \int_{0}^{t} \mathrm{~d} t^{\prime} 2 \lambda \cos \left(\omega t^{\prime}\right)}=T J_{0}\left(\frac{2 \lambda}{\hbar \omega}\right),
$$

we find that the Floquet Hamiltonian is

$$
H_{\mathrm{F}}=g_{1} \sigma^{x}+g_{2} J_{0}\left(\frac{2 \lambda}{\hbar \omega}\right) \sigma^{y} .
$$

The Bessel function $J_{0}(z) \rightarrow 1$ when $z \rightarrow 0$ and goes as $\sqrt{2 /(\pi z)} \cos (z-\pi / 4)$ when $z \rightarrow \infty$. It is clear that equations (72) and (75) are consistent with each other in the limit $\lambda /(\hbar \omega) \rightarrow 0$; in particular, $H_{\mathrm{F}}$ approaches the timeaveraged value $(1 / T) \int_{0}^{T} \mathrm{~d} t H(t)$ in the high-frequency limit. The limit $\lambda /(\hbar \omega) \rightarrow \infty$ is less trivial; we then see that the driving-dependent term in $H_{\mathrm{F}}$ goes to zero as $\sqrt{\hbar \omega / \lambda}$ apart from an oscillatory factor. Incidentally, we note that if we shift the time, i.e. change $\cos (\omega t) \rightarrow \cos \left(\omega\left(t+t_{0}\right)\right)$ in equation (72), the expression for the Floquet Hamiltonian in equation (75) would change.

Note that if we had considered a different limit where $\hbar \omega \gg$ $\lambda, g$, and used the FM expansion, we would have obtained an expansion in powers of $\lambda /(\hbar \omega)$ and $g /(\hbar \omega)$. A resummation of all the terms which are of first order in $g$ would then give back the expression in equation (75). Thus first-order FPT gives an expression for $H_{\mathrm{F}}$ which is a resummation of all the terms in the FM expansion which are of first order in the perturbation $V$.

Next, we apply FPT to a periodically driven spin chain called the PXP model [62]. We consider $H(t)=H_{0}(t)+V$, where

$$
\begin{aligned}
H_{0}(t) & =\frac{\lambda(t)}{2} \sum_{l} \sigma_{l}^{z}, \\
V & =g \sum_{l} P_{l-1} \sigma_{l}^{x} P_{l+1},
\end{aligned}
$$

where $P_{l}=\left(1-\sigma_{l}^{z}\right) / 2$ is the projection operator to the spindown state at site $l$. The presence of the projection operator in the second line of equation (76) makes the Hamiltonian $V$ non-integrable. We will consider a driving with the form of a square pulse,

$$
\begin{aligned}
\lambda(t)=-\lambda & \text { for } 0<t<T / 2 \\
& =+\lambda \quad \text { for } T / 2<t<T
\end{aligned}
$$

and $\lambda(t+T)=\lambda(t)$ for all times.

We now apply FPT assuming that $\lambda \gg g$. We choose the $\sigma_{l}^{z}$ basis for the states. According to the unperturbed Hamiltonian $H_{0}$ in equation (76), we see that such states $|n\rangle$ have an instantaneous energy eigenvalue $E_{n}(t)=(\lambda(t) / 2) \sum_{l} \sigma_{l}^{z}$. We now consider the effect to first order of the perturbation $V$ in equation (76). If $|m\rangle$ and $|n\rangle$ are two states which are connected by $V$, they differ by the value of $\sigma_{l}^{z}$ at only one site and therefore $E_{m}(t)-E_{n}(t)=\lambda(t)$. Hence equation (64) is satisfied and we have to use degenerate perturbation theory. The integral in 
equation (66) is found to be

$$
\int_{0}^{T} \mathrm{~d} t \mathrm{e}^{(\mathrm{i} / \hbar) \int_{0}^{t} \mathrm{~d} t^{\prime}\left[E_{m}\left(t^{\prime}\right)-E_{n}\left(t^{\prime}\right)\right]}=\frac{\mathrm{i} 2 \hbar}{\lambda}\left(\mathrm{e}^{-\mathrm{i} \lambda T /(2 \hbar)}-1\right) .
$$

We see that if $\mathrm{e}^{-\mathrm{i} \lambda T /(2 \hbar)}=1$, i.e. if

$$
\frac{\lambda}{\hbar \omega}=2 p,
$$

where $p$ is an integer, then the expression in equation (78) vanishes. This means that even in degenerate perturbation theory, there is no change in the Floquet eigenvalues and they remain equal to 1 .

We can now use equations (71) and (78) to derive the Floquet Hamiltonian. If $|m\rangle$ and $|n\rangle$ are two states which are connected by the perturbation $V$, we have $\langle m|V| n\rangle=g$ (note that $|m\rangle$ and $|n\rangle$ must necessarily be different from each other). We then obtain

$$
\begin{aligned}
\left(H_{\mathrm{F}}\right)_{m n} & =\frac{\mathrm{i} 2 g \hbar}{\lambda T}\left(\mathrm{e}^{-\mathrm{i} \lambda T /(2 \hbar)}-1\right) \\
& =\frac{2 g \hbar \omega}{\pi \lambda} \mathrm{e}^{-\mathrm{i} \pi \lambda /(2 \hbar \omega)} \sin \left(\frac{\pi \lambda}{2 \hbar \omega}\right) .
\end{aligned}
$$

We thus see that

$$
H_{\mathrm{F}}=g \frac{\sin \gamma}{\gamma} \sum_{l} P_{l-1}\left[\cos \gamma \sigma_{l}^{x}+\sin \gamma \sigma_{l}^{y}\right] P_{l+1},
$$

where $\gamma=\pi \lambda /(2 \hbar \omega)$. We now see that if equation (79) holds, the Floquet Hamiltonian vanishes to first order in $g / \lambda$. We then have to go to higher orders or study the model numerically to understand its behavior [62].

Equation (81) shows that in the limit $\lambda \rightarrow \infty, H_{\mathrm{F}}$ goes to zero as $1 / \lambda$ apart from some oscillatory factors. The different power laws, $1 / \sqrt{\lambda}$ versus $1 / \lambda$, in equations (75) and (81) are related to the fact that the driving term has different forms in the two cases, $\lambda \cos (\omega t)$ in the first case and the square pulse in equation (77) in the second case.

Finally, we consider a case where the Floquet Hamiltonian $H_{\mathrm{F}}$ has no contributions to first order in the perturbation and we have to go up to second order. Further, we will take the periodically driven part of the Hamiltonian to be much smaller than the time-independent part, and we will calculate $H_{\mathrm{F}}$ only within a particular sector of eigenstates of the timeindependent part. We consider a system with $M$ states $|\alpha\rangle$ in sector 1 , all of which have energy $E_{1}$, and $N$ states $|\beta\rangle$ in sector 2 , all of which have energy $E_{2}$. We introduce a small timedependent coupling between states in the two sectors given by $V(t)$ and its Hermitian conjugate $V^{\dagger}(t)$ as follows, where $V(t+T)=V(t)$. Denoting states in sectors 1 and 2 by $\Psi_{1}$ and $\Psi_{2}$, which are columns with $M$ and $N$ entries respectively, the Schrödinger equation takes the form

$$
\begin{aligned}
& \mathrm{i} \hbar \frac{\partial \Psi_{1}}{\partial t}=E_{1} \Psi_{1}+V \Psi_{2}, \\
& \mathrm{i} \hbar \frac{\partial \Psi_{2}}{\partial t}=E_{2} \Psi_{2}+V^{\dagger} \Psi_{1},
\end{aligned}
$$

where $V$ is an $M \times N$ dimensional matrix. We now look for a Floquet eigenstate $\psi(t)$ which lies mainly in sector 1 , namely,

$$
\begin{aligned}
\psi(t)= & \left(\sum_{\alpha} c_{\alpha}(t)|\alpha\rangle\right) \mathrm{e}^{-\mathrm{i} E_{1} t / \hbar} \\
& +\left(\sum_{\beta} c_{\beta}(t)|\beta\rangle\right) \mathrm{e}^{-\mathrm{i} E_{2} t / \hbar},
\end{aligned}
$$

where $c_{\alpha}(t)$ are of order 1 while $c_{\beta}(t)$ are of order $V$. Within sector $1, H_{\mathrm{F}}$ will be equal to $E_{1}$ plus terms of order $V^{2}$ (there are no contributions to first order in $V$ since the driving term has no matrix elements within sector 1 ). To calculate $H_{\mathrm{F}}$, we proceed as follows. Denoting the column of coefficients $c_{\alpha}$ and $c_{\beta}$ collectively as $c_{1}$ and $c_{2}$ respectively, we have

$$
\begin{aligned}
& \mathrm{i} \hbar \dot{c}_{1}=\mathrm{e}^{\mathrm{i}\left(E_{1}-E_{2}\right) t / \hbar} V c_{2}, \\
& \mathrm{i} \hbar \dot{c}_{2}=\mathrm{e}^{\mathrm{i}\left(E_{2}-E_{1}\right) t / \hbar} V^{\dagger} c_{1} .
\end{aligned}
$$

In the second equation in equation (84), we set $c_{1}(t)=c_{1}(0)$ on the right-hand side since we want to find $c_{2}(t)$ only to order $V$. Integrating in time, we obtain

$$
c_{2}(t)-c_{2}(0)=-\frac{\mathrm{i}}{\hbar} \int_{0}^{t} \mathrm{~d} t^{\prime} \mathrm{e}^{\mathrm{i}\left(E_{2}-E_{1}\right) t^{\prime} / \hbar} V^{\dagger}\left(t^{\prime}\right) c_{1}(0) .
$$

Since the Floquet eigenvalue in sector 1 is $\mathrm{e}^{-\mathrm{i} E_{1} t / \hbar}$ to first order in $V$, we require

$$
c_{1}(T)=c_{1}(0) \quad \text { and } \quad c_{2}(T)=\mathrm{e}^{\mathrm{i}\left(E_{2}-E_{1}\right) t / \hbar} c_{2}(0)
$$

to first order. Using this, we find from equation (85) that

$$
\begin{aligned}
c_{2}(t)= & -\frac{\mathrm{i}}{\hbar}\left[\frac{\int_{0}^{T} \mathrm{~d} t^{\prime} \mathrm{e}^{\mathrm{i}\left(E_{2}-E_{1}\right) t^{\prime} / \hbar} V^{\dagger}\left(t^{\prime}\right)}{\mathrm{e}^{\mathrm{i}\left(E_{2}-E_{1}\right) T / \hbar}-1}\right. \\
& \left.+\int_{0}^{t} \mathrm{~d} t^{\prime} \mathrm{e}^{\mathrm{i}\left(E_{2}-E_{1}\right) t^{\prime} / \hbar} V^{\dagger}\left(t^{\prime}\right)\right] c_{1}(0) .
\end{aligned}
$$

The first equation in equation (84), then gives

$$
c_{1}(T)=c_{1}(0)-\frac{\mathrm{i}}{\hbar} \int_{0}^{T} \mathrm{~d} t \mathrm{e}^{\mathrm{i}\left(E_{1}-E_{2}\right) t / \hbar} V(t) c_{2}(t) .
$$

Since the Floquet Hamiltonian in sector 1 satisfies $\psi_{1}(T)=$ $\mathrm{e}^{-\mathrm{i} H_{\mathrm{F}} T / \hbar} \psi_{1}(0)$, we see from equation (88) that

$$
\begin{aligned}
H_{\mathrm{F}}= & E_{1} I-\frac{\mathrm{i}}{\hbar T} \int_{0}^{T} \mathrm{~d} t \mathrm{e}^{\mathrm{i}\left(E_{1}-E_{2}\right) t / \hbar} V(t) \\
& \times\left[\frac{\int_{0}^{T} \mathrm{~d} t^{\prime} \mathrm{e}^{\mathrm{i}\left(E_{2}-E_{1}\right) t^{\prime} / \hbar} V^{\dagger}\left(t^{\prime}\right)}{\mathrm{e}^{\mathrm{i}\left(E_{2}-E_{1}\right) T / \hbar}-1}\right. \\
& \left.+\int_{0}^{t} \mathrm{~d} t^{\prime} \mathrm{e}^{\mathrm{i}\left(E_{2}-E_{1}\right) t^{\prime} / \hbar} V^{\dagger}\left(t^{\prime}\right)\right] .
\end{aligned}
$$

Next, the time periodicity of $V$ allows us to write it as

$$
V=\sum_{n=-\infty}^{\infty} V_{n} \mathrm{e}^{-\mathrm{i} n \omega t}
$$


Equation (89) then gives

$$
H_{\mathrm{F}}=E_{1} I-\sum_{n=-\infty}^{\infty} \frac{V_{n} V_{n}^{\dagger}}{E_{2}-E_{1}+n \hbar \omega} .
$$

Equation (91) shows that resonances occur whenever $\left(E_{2}-\right.$ $\left.E_{1}\right) /(\hbar \omega)$ is equal to an integer. However, near these points the above derivation of $H_{\mathrm{F}}$ breaks down since the Floquet eigenstates will no longer have $c_{2}(t)$ much smaller than $c_{1}(t)$, which was an assumption made in order to calculate $H_{\mathrm{F}}$. We note in passing that under a time shift $t \rightarrow t+t_{0}$, we would have $V_{n} \rightarrow V_{n} \mathrm{e}^{-\mathrm{i} n \omega t_{0}}$ in equation (90), but the Floquet Hamiltonian in equation (91) would not change.

As an example of the above formalism, we consider the Hubbard model with two sites, labeled 1 and 2, where the hopping amplitude between the two sites, $g$, is much smaller than the on-site interaction strength $U$ [82]. We will take the phase of the hopping to be a sinusoidal function of time; this describes the effect of a periodically varying electric field through the Peierls prescription (see section 5.1). The Hamiltonian is

$$
\begin{aligned}
H= & U \sum_{n=1,2} c_{n \uparrow}^{\dagger} c_{n \uparrow} c_{n \downarrow}^{\dagger} c_{n \downarrow} \\
& -g \sum_{\sigma=\uparrow, \downarrow}\left(\mathrm{e}^{\frac{\mathrm{i} a}{\omega} \sin (\omega t)} c_{1 \sigma}^{\dagger} c_{2 \sigma}\right. \\
& \left.+\mathrm{e}^{-\frac{\mathrm{i} a}{\omega} \sin (\omega t)} c_{2 \sigma}^{\dagger} c_{1 \sigma}\right) .
\end{aligned}
$$

We will consider a half-filled system with two electrons. In the undriven system $(a=0)$, we know that the low-energy states are described by an effective spin Hamiltonian given by $\left(4 g^{2} / U\right)\left(\vec{S}_{1} \cdot \vec{S}_{2} / \hbar^{2}-1 / 4\right)$. We will study what effect the driving has on the effective Hamiltonian which will now be denoted by $H_{\mathrm{F}}$.

In the space of two-electron states, the states

$$
|1 \uparrow, 2 \uparrow\rangle=c_{1 \uparrow}^{\dagger} c_{2 \uparrow}^{\dagger}|0\rangle \quad \text { and } \quad|1 \downarrow, 2 \downarrow\rangle=c_{1 \downarrow}^{\dagger} c_{2 \downarrow}^{\dagger}|0\rangle
$$

have a trivial dynamics since $H$ annihilates these states. Hence they are both Floquet eigenstates with Floquet eigenvalue equal to 1 . Next, we study the states in which there is one spin-up electron and one spin-down electron. There are four such states,

$$
\begin{aligned}
& |1\rangle=|1 \uparrow, 2 \downarrow\rangle=c_{1 \uparrow}^{\dagger} c_{2 \downarrow}^{\dagger}|0\rangle, \\
& |2\rangle=|1 \downarrow, 2 \uparrow\rangle=c_{1 \downarrow}^{\dagger} c_{2 \uparrow}^{\dagger}|0\rangle, \\
& |3\rangle=|1 \uparrow, 1 \downarrow\rangle=c_{1 \uparrow}^{\dagger} c_{1 \downarrow}^{\dagger}|0\rangle, \\
& |4\rangle=|2 \uparrow, 2 \downarrow\rangle=c_{2 \uparrow}^{\dagger} c_{2 \downarrow}^{\dagger}|0\rangle .
\end{aligned}
$$

In terms of equation (82), the first two states in equation (94) form sector 1 and have eigenvalues $E_{1}=0$ (low energy), the last two states form sector 2 and have eigenvalues $E_{2}=U$ (high energy), and the matrix $V$ relating the states of sector
2 to sector 1 is given by

$$
V=g\left(\begin{array}{cc}
-\mathrm{e}^{-\frac{\mathrm{i} a}{\omega} \sin (\omega t)} & -\mathrm{e}^{\frac{\mathrm{i} a}{\omega} \sin (\omega t)} \\
\mathrm{e}^{-\frac{\mathrm{i} a}{\omega} \sin (\omega t)} & \mathrm{e}^{\frac{\mathrm{i} a}{\omega} \sin (\omega t)}
\end{array}\right) .
$$

Using equation (91) and the identity $\mathrm{e}^{\mathrm{i} z \sin \phi}=$ $\sum_{n=-\infty}^{\infty} J_{n}(z) \mathrm{e}^{\mathrm{i} n \phi}$, where the Bessel functions satisfy $J_{n}(-z)=J_{-n}(z)=(-1)^{n} J_{n}(z)$, [81] we find that the Floquet Hamiltonian within sector 1 is

$$
H_{\mathrm{F}}=\left(\begin{array}{cc}
-1 & 1 \\
1 & -1
\end{array}\right) 2 g^{2} \sum_{n=-\infty}^{\infty} \frac{\left[J_{n}(a / \omega)\right]^{2}}{U+n \hbar \omega} .
$$

We see that one of the eigenstates of $H_{\mathrm{F}}$ is the state $(|1\rangle+$ $|2\rangle) / \sqrt{2}$ with eigenvalue zero (hence Floquet eigenvalue equal to 1); this is one of the three spin-triplet states, the other two being the ones given in equation (93). The other eigenstate of $H_{\mathrm{F}}$ is $(|1\rangle-|2\rangle) / \sqrt{2}$ which is a spin-singlet state, and the eigenvalue is $-4 g^{2} \sum_{n=-\infty}^{\infty}\left[J_{n}(a / \omega)\right]^{2} /(U+n \hbar \omega)$. Hence, in the spin language, the Floquet Hamiltonian has the form

$$
H_{\mathrm{F}}=4 g^{2} \sum_{n=-\infty}^{\infty} \frac{\left[J_{n}(a / \omega)\right]^{2}}{U+n \hbar \omega}\left(\frac{\vec{S}_{1} \cdot \vec{S}_{2}}{\hbar^{2}}-\frac{1}{4}\right) \text {. }
$$

We have so far discussed some ways of calculating the Floquet Hamiltonian perturbatively. We will now show that the Floquet unitary can also be calculated perturbatively [83]. Given a time-dependent Hamiltonian $H(t)$ (which may not commute with itself at different times), we define a timeevolution operator as

$$
U(t, 0)=\mathcal{T} \mathrm{e}^{-(\mathrm{i} / \hbar) \int_{0}^{t} \mathrm{~d} t H(t)} .
$$

Now suppose that $H(t)=H_{0}(t)+V$, where $H_{0}$ is timedependent but exactly solvable, and $V$ is a time-independent term which we want to treat perturbatively. We denote the time-evolution operator corresponding to $H_{0}$ as $U_{0}\left(t_{2}, t_{1}\right)$, so that

$$
\mathrm{i} \hbar \frac{\partial U_{0}(t, 0)}{\partial t}=H_{0}(t) U_{0}(t, 0) .
$$

Next, we define states in the interaction picture as

$$
\psi^{\mathrm{I}}(t)=U_{0}(0, t) \psi(t) .
$$

This satisfies the Schrödinger equation

$$
\mathrm{i} \hbar \frac{\partial \psi^{\mathrm{I}}}{\partial t}=V^{\mathrm{I}}(t) \psi^{\mathrm{I}}(t)
$$

where

$$
V^{\mathrm{I}}(t)=U_{0}(0, t) V U_{0}(t, 0) .
$$

The corresponding time-evolution operator

$$
U^{\mathrm{I}}(t, 0)=\mathcal{T} \mathrm{e}^{-(\mathrm{i} / \hbar) \int_{0}^{t} \mathrm{~d} t^{\prime} V^{\mathrm{I}}\left(t^{\prime}\right)}
$$

satisfies the equation

$$
\mathrm{i} \hbar \frac{\partial U^{\mathrm{I}}(t, 0)}{\partial t}=V^{\mathrm{I}}(t) U^{\mathrm{I}}(t, 0)
$$


Assuming the initial condition $U^{\mathrm{I}}(0,0)=I$, the solution of equation (104) is

$$
U^{\mathrm{I}}(t, 0)=I-\frac{\mathrm{i}}{\hbar} \int_{0}^{t} \mathrm{~d} t^{\prime} V^{\mathrm{I}}\left(t^{\prime}\right) U^{\mathrm{I}}\left(t^{\prime}, 0\right) .
$$

This provides an iterative way of calculating $U^{\mathrm{I}}(t, 0)$ in powers of $V^{\mathrm{I}}$. Thus one can write

$$
\begin{aligned}
U^{\mathrm{I}}(t, 0)= & I+\left(\frac{-\mathrm{i}}{\hbar}\right) \int_{0}^{t} \mathrm{~d} t^{\prime} V^{\mathrm{I}}\left(t^{\prime}\right) \\
& +\left(\frac{-\mathrm{i}}{\hbar}\right)^{2} \int_{0}^{t} \mathrm{~d} t_{1} V^{\mathrm{I}}\left(t_{1}\right) \int_{0}^{t_{1}} \mathrm{~d} t_{2} V^{\mathrm{I}}\left(t_{2}\right)+\cdots \\
= & I+U_{1}^{\mathrm{I}}(t, 0)+U_{2}^{\mathrm{I}}(t, 0)+\cdots,
\end{aligned}
$$

where the ellipsis denotes higher order terms. Finally, the full time-evolution operator is given by

$$
U(t, 0)=U_{0}(t, 0) U^{\mathrm{I}}(t, 0) .
$$

In the case where $U_{0}(T, 0)=I$, the Floquet operator is obtained by setting $t=T$ in equation (107) and is given by

$$
H_{\mathrm{F}}=\frac{\mathrm{i} \hbar}{T}\left[U_{1}^{\mathrm{I}}(T, 0)+\left(U_{2}^{\mathrm{I}}(T, 0)-\left(U_{1}^{\mathrm{I}}(T, 0)\right)^{2} / 2\right)+\cdots\right] .
$$

We end this section with a few comments regarding the FPT technique which deals directly with $U$. First, we note that the perturbation involving $U$ is useful if one attempts to compute higher order terms since it provides a straightforward and systematic way of obtaining such terms especially when $U_{0}(T, 0)=I$. This method has indeed been used to compute second and third order perturbative terms in several interacting many-body systems which is otherwise difficult $[38,84]$. Second, in contrast to the wave function method, the truncation of the perturbation series necessarily leads to loss of unitarity of $U$. In the case when $U_{0}(T, 0)=I$, this can be remedied by exponentiating the terms in $U^{\mathrm{I}}(T, 0)$. In contrast, such unitarization procedure is neither unique nor straightforward if $U_{0}(T, 0) \neq 0$. However, sometimes special dynamical symmetries of $U(T, 0)$ may help one to carry out the task [45].

\section{Other methods}

In this section, we present a brief discussion of three other methods which have been used in the literature to compute the Floquet Hamiltonian of a driven system.

\subsection{Rotating wave approximation}

The RWA provides a way to calculate an effective Hamiltonian by transforming to a 'rotating frame' in such a way that the Hamiltonian in this frame does not have any time-dependent terms to lowest order $[1,85]$. To see how this works, consider a time-dependent Hamiltonian $H(t)$ and a general wave function $\psi(t)$ satisfying the Schrödinger equation i $\hbar \partial \psi / \partial t=H \psi$.
Given a unitary operator $W(t)$ which transforms to a rotating frame, we define a wave function in that frame as

$$
\psi_{\mathrm{R}}(t)=W(t) \psi(t)
$$

We find that $\psi_{\mathrm{R}}$ satisfies the Schrödinger equation $\mathrm{i} \hbar \partial \psi_{\mathrm{R}} / \partial t=H_{\mathrm{R}} \psi_{\mathrm{R}}$, where

$$
H_{\mathrm{R}}=W H W^{-1}+\mathrm{i} \hbar \frac{\partial W}{\partial t} W^{-1}
$$

is the Hamiltonian in the rotating frame.

Now suppose that $H(t)=H_{0}(t)+V$, where $H_{0}(t+T)=$ $H_{0}(t)$. We can then try to choose $W(t)$ in such a way that the time-dependent part of $H_{\mathrm{R}}$ in equation (110) is as small as possible. For instance, if $H_{0}$ is much larger than $V$, and $H_{0}(t)$ commutes with itself at different times, we choose

$$
W(t)=\mathrm{e}^{(\mathrm{i} / \hbar) \int_{0}^{t} \mathrm{~d} t^{\prime} H_{0}\left(t^{\prime}\right)} .
$$

Since $W$ and $H_{0}$ commute with each other at different times, we see from equations (110) and (111) that

$$
H_{\mathrm{R}}=W V W^{-1},
$$

which implies that the large term $H_{0}$ has disappeared in going from $H$ to $H_{\mathrm{R}}$.

We would like $W$ to satisfy $W(t+T)=W(t)$. Equation (111) implies that this will be true if $H_{0}(t)$ has a complete set of orthonormal eigenstates $|n\rangle$ with eigenvalue $E_{n}(t)$, such that

$$
\int_{0}^{T} \mathrm{~d} t E_{n}(t)=0
$$

for all $n$; this turns out to be true in many problems. Then $H_{\mathrm{R}}$ in equation (112) will satisfy $H_{\mathrm{R}}(t+T)=H_{\mathrm{R}}(t)$. Equation (113) also implies equation (64) which means that we have to do degenerate FPT.

Next, inserting the identity operator, $I=\sum_{n}|n\rangle\langle n|$, on the left and right sides of $H_{\mathrm{R}}$ in equation (112), we obtain

$$
H_{\mathrm{R}}(t)=\sum_{m, n}|m\rangle\langle m|V| n\rangle\langle n| \mathrm{e}^{(\mathrm{i} / \hbar) \int_{0}^{t} \mathrm{~d} t^{\prime}\left[E_{m}\left(t^{\prime}\right)-E_{n}\left(t^{\prime}\right)\right]} .
$$

If we now do an FM expansion with $H_{\mathrm{R}}$, the first term is

$$
\begin{aligned}
H_{M}^{(0)}= & \frac{1}{T} \int_{0}^{T} \mathrm{~d} t H_{\mathrm{R}}(t) \\
= & \sum_{m, n}|m\rangle\langle n| \frac{\langle m|V| n\rangle}{T} \\
& \times \int_{0}^{T} \mathrm{~d} t \mathrm{e}^{(\mathrm{i} / \hbar) \int_{0}^{t} \mathrm{~d} t^{\prime}\left[E_{m}\left(t^{\prime}\right)-E_{n}\left(t^{\prime}\right)\right]} .
\end{aligned}
$$

The matrix elements of $H_{\mathrm{M}}^{(0)}$ in equation (115) agree with those of the Floquet Hamiltonian $H_{\mathrm{F}}$ given in equation (71). (Note that the second line of equation (71) vanishes due to the condition in equation (113).) We thus see that there is a connection between FPT and RWA if the periodically driven term is the one with the largest coefficient. However, the higher order terms that we get in the FM expansion of $H_{\mathrm{R}}(t)$ have no counterparts in $H_{\mathrm{F}}$ obtained from FPT. 
A simple use of the RWA is to solve the problem of a spin$1 / 2$ particle in a magnetic field which is rotating about one axis [1]. We consider the Hamiltonian

$$
H=B_{3} \sigma^{z}+B_{1}\left[\cos (\omega t) \sigma^{x}+\sin (\omega t) \sigma^{y}\right] .
$$

Using equation (110), we find that the operator

$$
W=\mathrm{e}^{\mathrm{i} \omega t / 2} \mathrm{e}^{\mathrm{i}(\omega t / 2) \sigma^{z}}
$$

which rotates by an angle $\omega t$ around the $\widehat{z}$-axis gives the Hamiltonian

$$
H_{\mathrm{R}}=\left(B_{3}-\frac{\hbar \omega}{2}\right) \sigma^{z}+B_{1} \sigma^{x}-\frac{\hbar \omega}{2} I
$$

(The factor of $\mathrm{e}^{\mathrm{i} \omega t / 2}$ has been included in equation (117) to ensure that $W(t+T)=W(t)$.) We see that $H_{\mathrm{R}}$ is completely time-independent and has the eigenvalues

$$
E_{ \pm}=-\frac{\hbar \omega}{2} \pm \sqrt{\left(B_{3}-\frac{\hbar \omega}{2}\right)^{2}+B_{1}^{2}} .
$$

We can use the eigenvalues $E_{ \pm}$and the corresponding orthonormal eigenstates $\psi_{ \pm}$of $H_{\mathrm{R}}$ to find the general solution for $\psi(t)$ through equation (109).

No assumptions were made about the relative magnitudes of $\hbar \omega, B_{3}$ and $B_{1}$ while deriving equation (118). We now note that when $\omega \rightarrow \infty, H_{\mathrm{R}}$ diverges instead of approaching the time-averaged value $(1 / T) \int_{0}^{T} \mathrm{~d} t H=B_{3} \sigma^{z}$ which is finite. This can be fixed as follows [1]. An examination of equation (119) shows that if $\omega \rightarrow \infty, E_{+} \rightarrow-B_{3}$ and $E_{-} \rightarrow B_{3}-\hbar \omega$, where we have ignored terms of order $1 / \omega$; in the same limit, $\psi_{+} \rightarrow$ $(0,1)$ and $\psi_{-} \rightarrow(1,0)$. Since the Floquet eigenvalues $\mathrm{e}^{-\mathrm{i} E_{ \pm} T / \hbar}$ remain invariant if the quasienergies $E_{ \pm}$are shifted by arbitrary integer multiples of $\hbar \omega$, we use this freedom to add $\hbar \omega$ to $E_{-}$while keeping $E_{+}$as it is. This gives us new quasienergies

$$
E_{ \pm}^{\prime}= \pm\left[-\frac{\hbar \omega}{2}+\sqrt{\left(B_{3}-\frac{\hbar \omega}{2}\right)^{2}+B_{1}^{2}}\right]
$$

which tend to $\mp B_{3}$ as $\omega \rightarrow \infty$. Combining these with the eigenstates $\psi_{ \pm}$of $H_{\mathrm{R}}$ in equation (118), we construct a new Hamiltonian $H_{\mathrm{R}}^{\prime}=E_{+}^{\prime} \psi_{+} \psi_{+}^{\dagger}+E_{-}^{\prime} \psi_{-} \psi_{-}^{\dagger}$ which gives

$$
\begin{aligned}
H_{\mathrm{R}}^{\prime}= & \frac{-\frac{\hbar \omega}{2}+\sqrt{\left(B_{3}-\frac{\hbar \omega}{2}\right)^{2}+B_{1}^{2}}}{\sqrt{\left(B_{3}-\frac{\hbar \omega}{2}\right)^{2}+B_{1}^{2}}} \\
& \times\left[\left(B_{3}-\frac{\hbar \omega}{2}\right) \sigma^{z}+B_{1} \sigma^{x}\right] .
\end{aligned}
$$

In the limit $\omega \rightarrow \infty, H_{\mathrm{R}}^{\prime} \rightarrow B_{3} \sigma^{z}$ as desired.

In the problem discussed above, the RWA expression for the quasienergies given in equation (120) agrees exactly with the quasienergies that we would have got from the Floquet operator $U(T, 0)$ if we had not used the RWA. (This is because the operator $W$ we used in equation (117) to perform the
RWA satisfies $W(T)=W(0)=I$, and, further, the Hamiltonian $H_{\mathrm{R}}$ that we obtained in equation (118) is completely timeindependent.) Now suppose that we have a Hamiltonian which differs slightly from the one in equation (116), namely,

$$
H=B_{3} \sigma^{z}+B_{1} \cos (\omega t) \sigma^{x}+B_{2} \sin (\omega t) \sigma^{y},
$$

where $\left|B_{1}-B_{2}\right|$ is not zero but is much smaller than $B_{1}$. Then performing the RWA with the same operator as in equation (117), we obtain the Hamiltonian

$$
\begin{aligned}
H_{\mathrm{R}}= & \left(B_{3}-\frac{\hbar \omega}{2}\right) \sigma^{z}+\frac{1}{2}\left(B_{1}+B_{2}\right) \sigma^{x}-\frac{\hbar \omega}{2} I \\
& +\frac{1}{2}\left(B_{1}-B_{2}\right)\left[\cos (2 \omega t) \sigma^{x}-\sin (2 \omega t) \sigma^{y}\right] .
\end{aligned}
$$

We see that this Hamiltonian has a time-dependent term but it is small. To lowest order in the RWA, we can ignore this term which leaves us with

$$
H_{\mathrm{R}}=\left(B_{3}-\frac{\hbar \omega}{2}\right) \sigma^{z}+\frac{1}{2}\left(B_{1}+B_{2}\right) \sigma^{x}-\frac{\hbar \omega}{2} I .
$$

We can then proceed in the same way as in equations (119)-(121) to obtain a final Hamiltonian which, in the limit $\omega \rightarrow \infty$, again gives $H_{\mathrm{R}}^{\prime} \rightarrow B_{3} \sigma^{z}$.

We now present another application of the RWA. We consider a tight-binding model of spinless particles (which may be either fermions or bosons) in one dimension placed in an electric field $\mathcal{E}(t)$ which varies in time with a period $T$ [85]. If the spacing between neighboring sites is $d$, the electric field can be put in as an on-site potential $-q n d \mathcal{E}(t)$ at site $n$, where $q$ is the charge of the particle. The complete Hamiltonian is

$$
H=\sum_{n=-\infty}^{\infty}\left[-g\left(c_{n}^{\dagger} c_{n+1}+c_{n+1}^{\dagger} c_{n}\right)-q d \mathcal{E}(t) n c_{n}^{\dagger} c_{n}\right]
$$

We can now eliminate the on-site potential in equation (125) and move the time-dependence to the phases of the nearestneighbor hoppings by transforming with

$$
W(t)=\mathrm{e}^{-(\mathrm{i} q d / \hbar) \int_{0}^{t} \mathrm{~d} t^{\prime} \mathcal{E}\left(t^{\prime}\right) \sum_{n=-\infty}^{\infty} n c_{n}^{\dagger} c_{n}} .
$$

Using equation (110), we find that the Hamiltonian in the rotating frame is

$$
\begin{aligned}
H_{R}= & -g \sum_{n=-\infty}^{\infty}\left[\mathrm{e}^{(\mathrm{i} q d / \hbar) \int_{0}^{t} \mathrm{~d} t^{\prime} \mathcal{E}\left(t^{\prime}\right)} c_{n}^{\dagger} c_{n+1}\right. \\
& \left.+\mathrm{e}^{-(\mathrm{i} q d / \hbar) \int_{0}^{t} \mathrm{~d} t^{\prime} \mathcal{E}\left(t^{\prime}\right)} c_{n+1}^{\dagger} c_{n}\right]
\end{aligned}
$$

(We note that the transformation from equations (125) to (127) is a gauge transformation which takes us from an electrostatic potential defined at a site $n$ to a vector potential which appears in the phase of the hopping between sites $n$ and $n+1$ according to the Peierls prescription.) Next, we define

$$
f(t)=\frac{q d}{\hbar} \int_{0}^{t} \mathrm{~d} t^{\prime} \mathcal{E}\left(t^{\prime}\right)
$$


Let us assume that $\int_{0}^{T} \mathrm{~d} t \mathcal{E}(t)=0$; then the periodicity of $\mathcal{E}(t)$ also implies the periodicity of $f(t)$ and therefore of $\mathrm{e}^{\mathrm{i} f(t)}$. We then find, by going to momentum space, that equation (127) takes the form

$$
H_{\mathrm{R}}=\int_{-\pi / d}^{\pi / d} \frac{d k}{2 \pi / d}[-2 g \cos (k+f(t))] c_{k}^{\dagger} c_{k} .
$$

Now suppose that $\mathrm{e}^{\mathrm{i} f}$ has the Fourier expansion

$$
\mathrm{e}^{\mathrm{i} f(t)}=\sum_{m=-\infty}^{\infty} F_{m} \mathrm{e}^{-\mathrm{i} m \omega t}
$$

so that $(1 / T) \int_{0}^{T} \mathrm{~d} t \mathrm{e}^{\mathrm{i} f(t)}=F_{0}$. If we now do an FM expansion of the Hamiltonian in equation (129), we find that only the first term given by $H_{\mathrm{M}}=(1 / T) \int_{0}^{T} \mathrm{~d} t H_{\mathrm{R}}$ survives. This is because if the Fourier expansion of the Hamiltonian in equation (129) is given by

$$
H_{\mathrm{R}}=\sum_{m=-\infty}^{\infty} H_{m} \mathrm{e}^{-\mathrm{i} m \omega t},
$$

we see that each of the Fourier components $H_{m}$ is diagonal in the momentum basis, and therefore they all commute with each other. Hence only the first term in the FM expansion is nonvanishing since all the higher order terms involve commutators of different $H_{m}$ 's [79]. We therefore obtain

$$
\begin{aligned}
H_{\mathrm{M}} & =\int_{-\pi / d}^{\pi / d} \frac{d k}{2 \pi / d} E_{k} c_{k}^{\dagger} c_{k}, \\
E_{k} & =-g\left(F_{0} \mathrm{e}^{\mathrm{i} k}+F_{0}^{*} \mathrm{e}^{-\mathrm{i} k}\right) .
\end{aligned}
$$

As an example, if $(q d / \hbar) \mathcal{E}(t)=a \cos (\omega t)$, we have $F_{0}=J_{0}(a / \omega)$. The energy-momentum dispersion is then given by $E_{k}=-2 g J_{0}(a / \omega) \cos k$, to be compared with the dispersion $-2 g \cos k$ for the undriven system. Note that a flat band is generated if $a$ and $\omega$ satisfy $J_{0}(a / \omega)=0$, and this leads to the dynamical localization of any wave packet since the group velocity $\mathrm{d} E_{k} / \mathrm{d} k=0$ for all values of $k$.

\subsection{Floquet-Magnus resummation}

For periodically kicked models where the Floquet unitary is of the form (assuming $\hbar=1$ )

$$
U(T)=\exp (-\mathrm{i} T H) \exp (-\mathrm{i} \epsilon V)=U_{0} \exp (-\mathrm{i} \epsilon V),
$$

where $[H, V] \neq 0$ and $V$ represents a periodic kicking term (e.g. see equation (15)) with $\epsilon \ll 1$ being a small parameter, a replica trick can be used [86] to write the Floquet Hamiltonian as a power series in $\epsilon$ instead of using the standard $\mathrm{BCH}$ formula (equation (12)) which yields a power series in $T$. Closed-form expressions for $H_{\mathrm{F}}$ can then be computed in some cases [86-88]. Using the replica trick,

$$
\begin{aligned}
T H_{\mathrm{F}} & =\mathrm{i} \ln U(T) \\
& =\mathrm{i} \lim _{\rho \rightarrow 0} \frac{1}{\rho}\left(U^{\rho}(T)-1\right) .
\end{aligned}
$$

Using a Taylor expansion in $\epsilon$ then yields the expression

$$
\mathrm{i} \ln U(T)=\mathrm{i} \lim _{\rho \rightarrow 0} \frac{1}{\rho}\left(\sum_{r=0}^{\infty}\left[\left.\frac{1}{r !}\left(\partial_{\epsilon}^{r} U^{\rho}(T)\right)\right|_{\epsilon=0}\right] \epsilon^{r}\right)
$$

Interchanging the sum and limit in equation (135) then gives the power series

$$
\begin{aligned}
& H_{\mathrm{F}}=\frac{1}{T} \sum_{r=0}^{\infty} \Gamma_{r} \epsilon^{r}, \quad \text { where } \\
& \Gamma_{r}=\frac{\mathrm{i}}{r !} \lim _{\rho \rightarrow 0} \frac{1}{\rho}\left[\left.\left(\partial_{\epsilon}^{r} U^{\rho}(T)\right)\right|_{\epsilon=0}\right] .
\end{aligned}
$$

So far the discussion was general, and further progress in computing $\Gamma_{r}$ is made by restricting to $U(T)$ of the form shown in equation (133). The general expression for $\Gamma_{r}$ is worked out in reference [86]. We restrict ourselves to the $\mathrm{O}(\epsilon)$ term which only requires $\Gamma_{1}$ that can be obtained straightforwardly (assuming an integer replica index $\rho$ ):

$$
\Gamma_{1}=\mathrm{i} \lim _{\rho \rightarrow 0} \frac{U_{0}^{\rho}}{\rho}\left[\sum_{m=0}^{\rho-1} U_{0}^{-m} V U_{0}^{m}\right] .
$$

The limit $\rho \rightarrow 0$ is then taken following an analytic continuation to arbitrary real values. We illustrate this using an example from reference [88] where in equation (133), we take a one-dimensional driven Ising model with

$$
\begin{aligned}
H & =\sum_{j}\left(J \sigma_{j}^{z} \sigma_{j+1}^{z}+h_{z} \sigma_{j}^{z}\right), \\
V(t) & =\sum_{n=-\infty}^{\infty} V \delta(t-n T), \quad V=h_{x} \sum_{j} \sigma_{j}^{x} .
\end{aligned}
$$

Since $H$ contains a sum of commuting terms, $U_{0}^{m}$ can be evaluated to finally give

$$
\begin{aligned}
U_{0}^{-m} V U_{0}^{m}= & -\frac{h_{x}}{2} \sum_{j} \sin (4 m J T)\left[\sigma _ { j - 1 } ^ { z } \left(\cos \left(2 m h_{z} T\right) \sigma_{j}^{y}\right.\right. \\
& \left.+\sin \left(2 m h_{z} T\right) \sigma_{j}^{x}\right) \\
& \left.+\left(\cos \left(2 m h_{z} T\right) \sigma_{j}^{y}+\sin \left(2 m h_{z} T\right) \sigma_{j}^{x}\right) \sigma_{j+1}^{z}\right] \\
& -h_{x} \sum_{j} \sin ^{2}(2 m J T) \sigma_{j-1}^{z} \\
& \times\left[\cos \left(2 m h_{z} T\right) \sigma_{j}^{x}-\sin \left(2 m h_{z} T\right) \sigma_{j}^{y}\right] \sigma_{j+1}^{z} \\
& +h_{x} \sum_{j} \cos ^{2}(2 m J T) \\
& \times\left[\cos \left(2 m h_{z} T\right) \sigma_{j}^{x}-\sin \left(2 m h_{z} T\right) \sigma_{j}^{y}\right] .
\end{aligned}
$$

To evaluate the sum over $m$ in equation (137), we use a mode expansion for $\sin (\theta)=(\exp (\mathrm{i} \theta)-\exp (-\mathrm{i} \theta)) /(2 \mathrm{i})$ and $\cos (\theta)=(\exp (i \theta)+\exp (-\mathrm{i} \theta)) / 2$ in equation (139), collect 
terms with different exponents, and use summations of geometric sequences to finally get

$$
\begin{aligned}
\sum_{m=0}^{\rho-1} U_{0}^{-m} V U_{0}^{m}= & -h_{x}\left[\frac { 1 } { 8 \mathrm { i } } \left(F_{4 J T-2 h_{z} T}^{-}(\rho)\right.\right. \\
& \left.+F_{4 J T+2 h_{z} T}^{-}(\rho)\right) \sum_{j}\left(\sigma_{j}^{z} \sigma_{j+1}^{y}+\sigma_{j}^{y} \sigma_{j+1}^{z}\right) \\
& +\frac{1}{8}\left(F_{4 J T-2 h_{z} T}^{+}(\rho)-F_{4 J T+2 h_{z} T}^{+}(\rho)\right) \\
& \times \sum_{j}\left(\sigma_{j}^{x} \sigma_{j+1}^{z}+\sigma_{j}^{z} \sigma_{j+1}^{x}\right) \\
& -\frac{1}{8}\left(F_{4 J T-2 h_{z} T}^{+}(\rho)+F_{4 J T+2 h_{z} T}^{+}(\rho)\right. \\
& \left.-2 F_{-2 h_{Z} T}^{+}(\rho)\right) \sum_{j}\left(\sigma_{j-1}^{z} \sigma_{j}^{x} \sigma_{j+1}^{z}\right) \\
& +\frac{\mathrm{i}}{8}\left(F_{4 J T-2 h_{z} T}^{-}(\rho)-F_{4 J T+2 h_{z} T}^{-}(\rho)\right. \\
& \left.-2 F_{-2 h_{Z} T}^{-}(\rho)\right) \sum_{j}\left(\sigma_{j-1}^{z} \sigma_{j}^{y} \sigma_{j+1}^{z}\right) \\
& \left.\left.+2 F_{-2 h_{Z} T}^{-}(\rho)\right) \sum_{j}\left(\sigma_{j}^{y}\right)\right] \\
& -\frac{1}{8}\left(F_{4 J T-2 h_{z} T}^{+}(\rho)-F_{4 J T+2 h_{z} T}^{+}(\rho)\right. \\
& \left.+2 F_{-2 h_{Z} T}^{+}(\rho)\right) \sum_{j}\left(\sigma_{j}^{x}\right) \\
& \frac{\mathrm{i}}{8}\left(F_{4 J T-2 h_{z} T}^{-}(\rho)-F_{4 J T+2 h_{z} T}^{-}(\rho)\right. \\
& \\
& \\
&
\end{aligned}
$$

where

$$
\begin{aligned}
& F_{\chi}^{-}(\rho)=-\mathrm{i} \frac{\cos (\chi \rho-\chi / 2)-\cos (\chi / 2)}{\sin (\chi / 2)}, \\
& F_{\chi}^{+}(\rho)=\frac{\sin (\chi \rho-\chi / 2)+\sin (\chi / 2)}{\sin (\chi / 2)} .
\end{aligned}
$$

Finally using equation (137) and taking the limit $\rho \rightarrow 0$, we get the expression for $H_{\mathrm{F}}$ to $\mathrm{O}(\epsilon)$,

$$
\begin{aligned}
H_{\mathrm{F}}= & \sum_{j}\left(J \sigma_{j}^{z} \sigma_{j+1}^{z}+h_{z} \sigma_{j}^{z}\right) \\
& +\epsilon h_{x}\left[J \sum_{j}\left(\sigma_{j}^{z} \sigma_{j+1}^{y}+\sigma_{j}^{y} \sigma_{j+1}^{z}\right)\right. \\
& +\frac{1}{4}\left[\left(2 J+h_{z}\right) \cot \left(2 J T+h_{z} T\right)\right. \\
& \left.-\left(2 J-h_{z}\right) \cot \left(2 J T-h_{z} T\right)\right] \sum_{j}\left(\sigma_{j}^{x} \sigma_{j+1}^{z}+\sigma_{j}^{z} \sigma_{j+1}^{x}\right) \\
& +\frac{1}{4}\left[\left(2 J-h_{z}\right) \cot \left(2 J T-h_{z} T\right)-\left(2 J+h_{z}\right)\right. \\
& \left.\times \cot \left(2 J T+h_{z} T\right)-2 h_{z} \cot \left(h_{z} T\right)\right]
\end{aligned}
$$

$$
\begin{aligned}
& \times \sum_{j}\left(\sigma_{j-1}^{z} \sigma_{j}^{x} \sigma_{j+1}^{z}\right) \\
& +\frac{1}{4}\left[\left(2 J-h_{z}\right) \cot \left(2 J T-h_{z} T\right)+\left(2 J+h_{z}\right)\right. \\
& \left.\times \cot \left(2 J T+h_{z} T\right)+2 h_{z} \cot \left(h_{z} T\right)\right] \sum_{j} \sigma_{j}^{x} \\
& \left.+h_{z} \sum_{j} \sigma_{j}^{y}\right]
\end{aligned}
$$

In reference [86], it was demonstrated for one-dimensional kicked Ising models that long prethermal Floquet regimes can exist in the thermodynamic limit even at intermediate and low drive frequencies that are governed by $H_{\mathrm{F}}$ obtained from the replica expansion. The thermalization of interacting systems has also been studied for the Hubbard model using nonequilibrium dynamical mean field theory [89]. It has been found that strong interactions lead to long-lived Floquet prethermal regimes; moreover, the existence of such regimes can be controlled by tuning the drive frequency.

\subsection{Hamiltonian flow method}

The Hamiltonian flow method was initially applied to timeindependent Hamiltonians by Wegner [90, 91]. The idea behind this method is to obtain an effective Hamiltonian, via generation of a flow, which is diagonal in a chosen basis (usually chosen to be the non-interacting single particle eigenstates). The flow is designed such that the off-diagonal terms reduce gradually as the flow continues. Such a flow is characterized by a parameter $\ell$ and is implemented via an antiunitary transformation $\eta(\ell)$. The flow equation, in terms of this anti-unitary operator $\eta$ is given by [90]

$$
\frac{\mathrm{d} H(\ell)}{\mathrm{d} \ell}=[\eta(\ell), H(\ell)]
$$

Of course, the key issue here is the choice of $\eta(\ell)$ and this depends on the system at hand. For example, for a two-level system with $H(\ell) \equiv H_{\mathrm{t}}$ given by

$$
H_{\mathrm{t}}=\sum_{i=1,2} \epsilon_{i}|i\rangle+\left(\Delta_{12}|1\rangle\langle 2|+\text { h.c. }\right) \text {, }
$$

it was shown that one can choose $\eta=-\left[H_{\mathrm{t}}, H_{\mathrm{D}}\right]$, where $H_{\mathrm{D}}$ is the diagonal part of $H_{\mathrm{t}}$. This choice leads to (taking $\Delta_{12}$ to be real for simplicity)

$$
\begin{aligned}
\frac{\partial \Delta_{12}}{\partial \ell} & =[\eta, H]_{12} \\
& =\sum_{j=1,2}\left(\epsilon_{1}+\epsilon_{2}-2 \epsilon_{j}\right) \Delta_{1 j} \Delta_{j 2} .
\end{aligned}
$$

where $\Delta_{11}=\epsilon_{1}$ and $\Delta_{22}=\epsilon_{2}$. It was shown in reference [90] that these equations lead to

$$
\Delta_{12}(\ell)=\Delta_{12}(\ell=0) \exp \left[-\left(\epsilon_{1}-\epsilon_{2}\right)^{2} \ell\right] .
$$


Thus the Hamiltonian reduces to a diagonal form with increasing $\ell$. The method gets more complicated with increasing number of energy levels, and in most cases the flow equations require a numerical solution.

As shown by several authors [92-94], this method can be modified to be applied to periodically driven systems. Here one tries to generate such a flow to compute the Floquet Hamiltonian. To this end, the Hamiltonian is divided into two parts, $H_{0}$ and $V$. Typically, $H_{0}$ is chosen to be the first term in the FM expansion of $H_{\mathrm{F}}$ so that

$$
H_{0}=\frac{1}{T} \int_{0}^{T} \mathrm{~d} t H(t), \quad V=H(t)-H_{0} .
$$

Note that $V \rightarrow 0$ for $T \rightarrow 0$, so that the method, for such a choice of the division of $H(t)$, is naturally accurate at high drive frequency. Next, the flow parameter $\ell$ and $\eta$ are introduced through the transformation $U=\exp (\eta[\ell, t])$, where

$$
\eta[\ell, t]=-\mathrm{i} \frac{\ell}{T} \int_{0}^{t} \mathrm{~d} t_{1} V\left[\ell, t_{1}\right]
$$

where we have extended the definition of $H(t)$ to $H[\ell, t]$ with

$$
V[\ell, t]=\frac{1}{T} \int_{0}^{T} \mathrm{~d} t_{1}\left(H[\ell, t]-H\left[\ell, t_{1}\right]\right) .
$$

Note that $V[0, t]=V(t)$ and our aim is show that $V[\infty, t]=0$.

To obtain the flow equation, one generates an infinitesimal change by $\delta \ell$ and relates $H[\ell+\delta \ell, t]$ to $H[\ell, t]$. A straightforward calculation charted out in reference [94] shows that this leads to the flow equation

$$
\frac{\mathrm{d} H[\ell, t]}{\mathrm{d} \ell}=-V[\ell, t]+\mathrm{i} \int_{0}^{t} \mathrm{~d} t_{1}[V[\ell, t], H[\ell, t]] .
$$

The general nature of the flow from this equation is quite complicated. However, consideration simplification occurs at high frequencies where the second term can be neglected. In this case the equation reduces to $\mathrm{d} V[\ell, t] / d \ell=-V[\ell, t]$ and leads to $V=V(t) \mathrm{e}^{-\ell}$. Thus $V[\infty, t]=0$ and $H=H_{0}$ (equation (147)) which is the standard FM result.

The existence of such fixed points of the flow equation at lower drive frequencies is not simple to prove. This issue and the application of the method to a simple spin model where the flow equations can be written down in a straightforward manner have been studied in reference [94]. However, the applicability of this method to more complicated interacting many-body systems and the fate of the flow equation at lower frequency remain interesting open problems.

Before ending this section, we note that a slightly different approach to the flow equation can be used to directly access the Floquet Hamiltonian. This method, used in reference [93], applies the canonical transformation used by Wegner [90] to Floquet quasienergies rather than the original Hamiltonian. The method has been applied to a disordered Anderson model (for which it is exact) and a driven many-body localized system in reference [93].

\section{Discussion}

In this review, we have aimed to provide a pedagogical discussion of several analytic methods for computing the Floquet Hamiltonian for interacting many-body systems. Out of these, the FM expansion method, the adiabatic-impulse approximation and the FPT are treated in detail. Three other methods, namely, the RWA, the FM resummation method, and the Hamiltonian flow technique have been discussed briefly.

The FM expansion is probably the most widely used perturbative method in the literature. This technique treats inverse of the drive frequency (in units of $J_{\text {loc }} / \hbar$, where $J_{\text {loc }}$ is a typical energy scale of the system) as the perturbation parameter and is therefore expected to be accurate in the high drive frequency regime. Moreover, it provides a perturbative expansion which maintains unitarity of $U$ at each order in perturbation theory. Also, the method has been quite successful in providing a qualitatively accurate picture of drive-induced generation of topologically non-trivial Floquet states and the presence of a long prethermal timescale in interacting many-body systems at high drive frequencies. The main weakness of this method is two-fold. First, the radius of convergence of the perturbation expansion and its regime of validity is difficult to determine. Second, the method may lead to qualitatively wrong pictures at intermediate and low drive frequencies [84]. The resolution of these issues which might provide one with a more complete picture of the FM expansion method is a long standing challenge; some progress in this direction has been made recently $[50,86]$.

In contrast, the adiabatic-impulse approximation leads to a method which is more accurate at low drive frequencies. This makes the method ideal for studying properties of driven systems in regimes where most other approximation methods fail. Moreover, the method provides access to the micromotion of the system; thus it allows one to obtain information about the phase bands which carries significantly more information about the properties of the driven system than the Floquet Hamiltonian. The key disadvantage of the method is that its application is limited to a class of integrable models; for more complicated non-integrable modes, it gets extremely complicated. The possibility of generalizing this method so that it may be applied to non-integrable and/or multi-band models is an open problem.

The FPT, in contrast to both the methods discussed above, does not treat the drive frequency or its inverse as a perturbation parameter. The perturbation parameter for this method is the ratio of amplitudes of the terms in the Hamiltonian; the largest term in the Hamiltonian (which consists of commuting terms) is treated exactly and the contribution of the rest, smaller, terms are assessed using perturbation theory. The method provides qualitatively accurate results even when the drive frequency is small compared to the amplitude of the largest term and can therefore access regimes where the FM expansion fails. When this largest amplitude term is also the term which implements the periodic drive, this method is analogous to the RWA to leading order. However, for other cases, the FPT leads to different, more accurate, results compared to 
the RWA [84]. Moreover, the first order contribution to the Floquet Hamiltonian in this method already constitutes a resummation of an infinite class of terms in the FM expansion; this has been explicitly demonstrated in the context of ultracold Rydberg atoms subjected to a square pulse protocol in reference [62]. The key disadvantages of this method are two-fold. First, it does not automatically lead to an unitary evolution operator at a given order in perturbation theory and a separate unitarization procedure is necessary. This procedure need not be unique specially when the zeroth order Floquet Hamiltonian, $H_{\mathrm{F}}^{(0)}$, does not vanish. Second, the method is difficult to apply if the largest amplitude term in the Hamiltonian is complicated so that its contribution to $U$ cannot be determined exactly. These issues constitute some open problems relevant for FPT.

Next, we note that we have not provided a discussion of numerical methods for computing $U$ or $H_{\mathrm{F}}$. This, in our opinion, warrants a separate review. Here we briefly comment that the standard method for this involves exact diagonalization (ED), specially when one wants an access to all states in the Hilbert space of the driven model necessary for tracking the dynamics at long times [95]. For this one proceeds as follows. First, one decomposes $U(T, 0)$ into a product of $N$ Trotter steps $\left(U_{j}=U\left(t_{j-1}+\delta t, t_{j-1}\right)\right.$ being the unitary at the jth step and $\delta t=T / N)$, where the value of $N$ depends on both the nature of the Hamiltonian and the drive protocol used. Here we note that $N$ is of order 1 if we use discrete protocols such as a square pulse or periodic kicks since for these protocols $H(T)$ remains constant for a large part of the drive cycle. In contrast, for continuous drives $N \gg 1$ and therefore such drive protocols are difficult to treat numerically. Next, one expresses $U_{j}$ using the eigenenergies and eigenvectors of $H_{j} \equiv H\left(t_{j-1}+\delta t / 2\right)$ which can be obtained using ED. Finally one computes the product over $U_{j}$ to construct $U$ and diagonalizes to obtain the Floquet eigenvectors and quasienergies. Clearly, the numerical cost of this method depends both on the Hilbert space dimension of the many-body system and $N$; thus this method is most useful for one-dimensional systems driven by discrete protocols. There are several other numerical methods for computing the Floquet spectrum of a driven many-body system [96], but we will not address them here.

We also note that quasiperiodically-driven many-body quantum systems, with two or more incommensurate drive frequencies, have received attention only recently [97-105]. While the steady state is again expected to be described by a featureless infinite temperature ensemble, much less is known about possible prethermal phases in such settings. In case such prethermal phases exist (see references [103] and [105]), they would likely constitute a much richer class than their Floquet analogs. Finding reliable perturbative approaches, particularly beyond the high-frequency regime where a generalization of the FM expansion to quasiperiodic drives exists [106], remains an uncharted territory.

Finally, we would like to point out that in this review we have not discussed path integrals methods for periodically driven quantum systems. It is well known that study of nonequilibrium dynamics using path integrals usually requires use of the Schwinger-Keldysh formalism [107]. This method is used for study of open systems [108], driven superconductors [109], and systems with dissipation treated within Lindblad approach [110]. It is also to be noted for closed integrable systems, path integral technique may be used for computation of the Floquet Hamiltonian of a periodically driven system for certain protocols without resorting to Keldysh techniques [111]. Overall, the path integral method is usually more widely used for treatment of open quantum systems and we do not study its details in this review. In addition, we have also omitted discussion of Floquet dynamical mean field theory which has been extensively covered in several works [112].

In conclusion we have compared and contrasted several analytic, albeit perturbative, techniques for computation of a periodically driven many-body system. Our review, while not entirely exhaustive, provides a pedagogical introduction to the technical details of several such methods and addresses some of the applications of these methods to a number of recent problems in periodically driven quantum many-body systems.

\section{Acknowledgments}

The work of AS is partly supported through the Max Planck Partner Group program between the Indian Association for the Cultivation of Science (Kolkata) and the Max Planck Institute for the Physics of Complex Systems (Dresden). DS thanks DST, India for Project No. SR/S2/JCB-44/2010 for financial support.

\section{Data availability statement}

All data that support the findings of this study are included within the article (and any supplementary files).

\section{ORCID iDs}

Arnab Sen (D) https://orcid.org/0000-0001-5342-8332

Diptiman Sen (10) https://orcid.org/0000-0002-6926-9230

K Sengupta (D) https://orcid.org/0000-0001-9679-3380

\section{References}

[1] Bukov M, D’Alessio L and Polkovnikov A 2015 Adv. Phys. 64 139

[2] D'Alessio L and Polkovnikov A 2013 Ann. Phys., NY 33319

[3] D'Alessio L, Kafri Y, Polkovnikov A and Rigol M 2016 Adv. Phys. 65239

[4] Shevchenko S N, Ashhab S and Nori F 2010 Phys. Rep. 4921

[5] Oka T and Kitamura S 2019 Annu. Rev. Condens. Matter Phys. 10387

[6] Blanes S, Casas F, Oteo J A and Ros J 2009 Phys. Rep. 470 151

[7] Dziarmaga J 2010 Adv. Phys. 591063

Polkovnikov A, Sengupta K, Silva A and Vengalattore M 2011 Rev. Mod. Phys. 83863

[8] Dutta A, Aeppli G, Chakrabarti B K, Divakaran U, Rosenbaum T F and Sen D 2015 Quantum Phase Transitions in Transverse Field Spin Models: From Statistical Physics to Quantum Information (Cambridge: Cambridge University Press)

Mondal S, Sen D and Sengupta K 2010 Quantum Quenching, Annealing and Computation (Lecture Notes in Physics) 
vol 802 ed A Das, A Chandra and B K Chakrabarti (Berlin: Springer) ch 2 p 21

De Grandi C and Polkovnikov A 2010 Quantum Quenching, Annealing and Computation (Lecture Notes in Physics) vol 802 ed A Das, A Chandra and B K Chakrabarti (Berlin: Springer) ch 6 p 75

[9] Bloch I, Dalibard J and Zwerger W 2008 Rev. Mod. Phys. 80 885

Taurell L and Sanchez-Palencia L 2018 C. R. Physique 19365

[10] Greiner M, Mandel O, Esslinger T, Hnsch T W and Bloch I 2002 Nature 41539

Orzel C, Tuchman A K, Fenselau M L, Yasuda M and Kasevich M A 2001 Science 2912386

Kinoshita T, Wenger T and Weiss D S 2006 Nature 440900

Saddler L E, Higbie J M, Leslie S R, Vengalattore M and Stamper-Kurn D M 2006 Nature $\mathbf{4 4 3} 312$

[11] Simon J, Bakr W S, Ma R, Tai M E, Preiss P M and Greiner M 2011 Nature $\mathbf{4 7 2} 307$

Bakr W S, Peng A, Tai M E, Ma R, Simon J, Gillen J I, Folling S, Pollet L and Greiner M 2010 Science 329547

[12] Bernien Het al 2017 Nature 551579

[13] Bluvstein D et al 2021 Science 3711355

[14] Ebadi S et al 2021 Nature 595227

[15] Kiefer P, Hakelberg F, Wittemer M, Bermúdez A, Porras D, Warring U and Schaetz T 2019 Phys. Rev. Lett. 123213605

[16] Lloyd S 1996 Science 2731073

Wallraff A, Schuster D I, Blais A, Frunzio L, Huang R S, Majer J, Kumar S, Girvin S M and Schoelkopf R J 2004 Nature 431 162

[17] Stepanov E A, Dutreix C and Katsnelson M I 2017 Phys. Rev. Lett. 118157201

[18] Floquet G 1883 Ann. Sci. Ecole Norm. Superieure 1247

[19] Kitagawa T, Berg E, Rudner M and Demler E 2010 Phys. Rev. B 82235114

Lindner N H, Refael G and Galitski V 2011 Nat. Phys. 7490

[20] Kitagawa T, Oka T, Brataas A, Fu L and Demler E 2011 Phys. Rev. B 84235108

Kundu A, Fertig H A and Seradjeh B 2014 Phys. Rev. Lett. 113 236803

[21] Thakurathi M, Patel A A, Sen D and Dutta A 2013 Phys. Rev. B 88155133

Thakurathi M, Sengupta K and Sen D 2015 Phys. Rev. B 89 235434

[22] Nathan F and Rudner M S 2015 New J. Phys. 17125014

[23] Carpentier D, Delplace P, Fruchart M and Gawedzki K 2015 Phys. Rev. Lett. 114106806

[24] Dutreix C, Stepanov E A and Katsnelson M I 2016 Phys. Rev. B $93241404(\mathrm{R})$

[25] Mukherjee B, Sen A, Sen D and Sengupta K 2016 Phys. Rev. B 94155122

Mukherjee B 2018 Phys. Rev. B 98235112

[26] Mukherjee B, Mohan P, Sen D and Sengupta K 2018 Phys. Rev. B 97205415

[27] Heyl M, Polkovnikov A and Kehrein S 2013 Phys. Rev. Lett. 110135704

For a review, see Heyl M 2018 Rep. Prog. Phys. 81054001

[28] Sen A, Nandy S and Sengupta K 2016 Phys. Rev. B 94214301 Nandy S, Sengupta K and Sen A 2018 J. Phys. A: Math. Theor. 51334002

[29] Oka T and Aoki H 2009 Phys. Rev. B 79 081406(R)

[30] Agarwala A, Bhattacharya U, Dutta A and Sen D 2016 Phys. Rev. B 93174301

[31] Dóra B, Cayssol J, Simon F and Moessner R 2012 Phys. Rev. Lett. 108056602

[32] Watanabe H and Oshikawa M 2015 Phys. Rev. Lett. 114251603

[33] Khemani V, Lazarides A, Moessner R and Sondhi S L 2016 Phys. Rev. Lett. 116250401

[34] Zhang J et al 2017 Nature 543217
[35] Else D V, Bauer B and Nayak C 2016 Phys. Rev. Lett. 117 090402

[36] Das A 2010 Phys. Rev. B 82172402

Bhattacharyya S, Das A and Dasgupta S 2012 Phys. Rev. B 86 054410

Hedge S S, Katiyar H, Mahesh T S and Das A 2014 Phys. Rev. B 90174407

[37] Mondal S, Pekker D and Sengupta K 2012 Europhys. Lett. 100 60007

Divakaran U and Sengupta K 2014 Phys. Rev. B 90184303

Kar S, Mukherjee B and Sengupta K 2016 Phys. Rev. B 94 075130

Lubini S, Chirondojan L, Oppo G, Politi A and Politi P 2019 Phys. Rev. Lett. 122084102

[38] Mukherjee B, Sen A, Sen D and Sengupta K 2020 Phys. Rev. B 102075123

[39] Haldar A, Sen D, Moessner R and Das A 2021 Phys. Rev. X 11 021008

[40] Nag T, Roy S, Dutta A and Sen D 2014 Phys. Rev. B 89 165425

Nag T, Sen D and Dutta A 2015 Phys. Rev. A 91063607

[41] Agarwala A and Sen D 2017 Phys. Rev. B 95014305

[42] Luitz D J, Bar Lev Y and Lazarides A 2017 SciPost Phys. 3 029

Luitz D J, Lazarides A and Bar Lev Y 2018 Phys. Rev. B 97 020303

[43] Wen X and Wu J-Q 2018 arXiv:1805.00031 unpublished

Wen X and Wu J-Q 2018 Phys. Rev. B 97184309

Fan R, Gu Y, Vishwanath A and Wen X 2020 Phys. Rev. X 10 031036

Wen X, Fan R, Vishwanath A and Gu Y 2021 Phys. Rev. Res. 3023044

Han B and Wen X 2020 Phys. Rev. B 102205125

Fan R, Gui Y, Vishwanath A and Wen X 2021 SciPost Phys. 10049

[44] Lapierre B, Choo K, Tauber C, Tiwari A, Neupert T and Chitra R 2020 Phys. Rev. Res. 2023085

Lapierre B, Choo K, Tiwari A, Tauber C, Neupert T and Chitra R 2020 Phys. Rev. Res. 2033461

[45] Das D, Ghosh R and Sengupta K 2021 J. High Energy Phys. JHEP05(2012)172

[46] Deutsch J M 1991 Phys. Rev. A 432046

Srednicki M 1994 Phys. Rev. E 50888

Srednicki M 1999 J. Phys. A: Math. Gen. 321163

[47] Lazarides A, Das A and Moessner R 2014 Phys. Rev. E 90 012110

[48] Ponte P, Chandran A, Papić Z and Abanin D A 2015 Ann. Phys., NY 353196

[49] D'Alessio L and Rigol M 2014 Phys. Rev. X 4041048

[50] Kuwahara T, Mori T and Saito K 2016 Ann. Phys., NY 36796

[51] Mori T, Kuwahara T and Saito K 2016 Phys. Rev. Lett. 116 120401

Bukov M, Gopalakrishnan S, Knap M and Demler E 2015 Phys. Rev. Lett. 115205301

Zeng T-S and Sheng D N 2017 Phys. Rev. B 96094202

Mori T 2018 Phys. Rev. B 98104303

Luitz D J, Moessner R, Sondhi S L and Khemani V 2020 Phys. Rev. X 10021046

Okamoto J and Perunaci F 2020 arXiv:2010.00326 unpublished

[52] Weidinger S A and Knap M 2017 Sci. Rep. 745382

Howell O, Weinberg P, Sels D, Polkovnikov A and Bukov M 2019 Phys. Rev. Lett. 122010602

Mizuta K, Takasan K and Kawakami N 2019 Phys. Rev. B 100 020301

Dalla Torre E G and Dentelski D 2020 arXiv:2005.07207 unpublished

[53] Singh K et al 2019 Phys. Rev. X 9041021 
Peng P, Yin C, Huang X, Ramanathan C and Cappellaro P 2021 Nat. Phys. 17444

Rubio-Abadal A, Ippoliti M, Hollerith S, Wei D, Rui J, Sondhi S L, Khemani V, Gross C and Bloch I 2020 Phys. Rev. X 10 021044

[54] Nandkishore R and Huse D A 2015 Annu. Rev. Condens. Matter Phys. 615

[55] Ponte P, Papić Z, Huveneers F and Abanin D A 2015 Phys. Rev. Lett. 114140401

Abanin D A, De Roeck W and Huveneers F 2016 Ann. Phys., NY 3721

[56] Rehn J, Lazarides A, Pollmann F and Moessner R 2016 Phys. Rev. B 94020201

Po H C, Fidkowski L, Morimoto T, Potter A C and Vishwanath A 2016 Phys. Rev. X 6041070

[57] Zhang L, Khemani V and Huse D A 2016 Phys. Rev. B 94 224202

Sonner M, Serbyn M, Papić Z and Abanin D A 2020 arXiv:2012.15676 unpublished

Chan A, De Luca A and Chalker J T 2021 Phys. Rev. Res. 3 023118

[58] Choi S, Turner C J, Pichler H, Ho W W, Michailidis A A, Papić Z, Serbyn M, Lukin M D and Abanin D A 2019 Phys. Rev. Lett. 122220603

Ho W W, Choi S, Pichler H and Lukin M D 2019 Phys. Rev. Lett. 122040603

[59] Turner C J, Michailidis A A, Abanin D A, Serbyn M and Papić Z 2018 Nat. Phys. 14745

Turner C J, Michailidis A A, Abanin D A, Serbyn M and Papić Z 2018 Phys. Rev. B 98155134

Bull K, Martin I and Papić Z 2019 Phys. Rev. Lett. 123 030601

[60] Khemani V, Laumann C R and Chandran A 2019 Phys. Rev. B 99161101

Maudgalya S, Regnault N and Bernevig B A 2018 Phys. Rev. B 98235156

Iadecola T, Schecter M and Xu S 2019 Phys. Rev. B 100 184312

Shiraishi N 2019 J. Stat. Mech. 083103

Schecter M and Iadecola T 2019 Phys. Rev. Lett. 123 147201

[61] Banerjee D and Sen A 2021 Phys. Rev. Lett. 126220601

[62] Mukherjee B, Nandy S, Sen A, Sen D and Sengupta K 2020 Phys. Rev. B 101245107

[63] Mizuta K, Takasan K and Kawakami N 2020 Phys. Rev. Res. 2 033284

Pai S and Pretko M 2019 Phys. Rev. Lett. 123136401

Sugiura S, Kuwahara T and Saito K 2021 Phys. Rev. Res. 3 012010

[64] Magnus W 1954 Commun. Pure Appl. Math. 7649

[65] Dutreix C and Katsnelson M I 2017 Phys. Rev. B 95024306

[66] Arnal A, Casas F and Chiralt C 2018 J. Phys. Commun. 2 035024

[67] Else D V, Bauer B and Nayak C 2017 Phys. Rev. X 7011026

[68] Abanin D A, De Roeck W, Ho W W and Huveneers F 2017 Phys. Rev. B 95014112

[69] Rodriguez-Vega M, Vogl M and Fiete G A 2021 Ann. Phys., NY 168434

[70] Child M S 1974 Molecular Collision Theory (London: Academic)

[71] Kayanuma Y 1994 Phys. Rev. A 50843

Kayanuma Y 1997 Phys. Rev. A 552495

[72] Sachdev S 1999 Quantum Phase Transitions (Cambridge: Cambridge University Press)

[73] Kitaev A 2006 Ann. Phys., NY 3212

Chen H-D and Nussinov Z 2008 J. Phys. A: Math. Theor. 41 075001

[74] Castro Neto A H, Guinea F, Peres N M R, Novoselov K S and Geim A K 2009 Rev. Mod. Phys. 81109
[75] Qi X-L and Zhang S-C 2011 Rev. Mod. Phys. 831057

[76] Armitage N P, Mele E J and Vishwanath A 2018 Rev. Mod. Phys. 90015001

[77] Sau J D and Sengupta K 2014 Phys. Rev. B 90104306

[78] Soori A and Sen D 2010 Phys. Rev. B 82115432

[79] Mikami T, Kitamura S, Yasuda K, Tsuji N, Oka T and Aoki H 2016 Phys. Rev. B 93144307

[80] Udupa A, Sengupta K and Sen D 2020 Phys. Rev. B 102 045419

[81] Abramowitz M and Stegun I A 1972 Handbook of Mathematical Functions (New York: Dover)

[82] Itin A P and Katsnelson M I 2015 Phys. Rev. Lett. 115 075301

[83] Bilitewski T and Cooper N R 2015 Phys. Rev. A 91033601

[84] Ghosh R, Mukherjee B and Sengupta K 2020 Phys. Rev. B 102 235114

Bukov M, Kolodrubetz M and Polkovnikov A 2016 Phys. Rev. Lett. 116125301

[85] Eckardt A 2017 Rev. Mod. Phys. 89011004

[86] Vajna S, Klobas K, Prosen T and Polkovnikov A 2018 Phys. Rev. Lett. 120200607

[87] Fleckenstein C and Bukov M 2021 Phys. Rev. B 103140302

[88] Fleckenstein C and Bukov M 2021 Phys. Rev. B 103 144307

[89] Peronaci F, Schiró M and Parcollet O 2018 Phys. Rev. Lett. 120 197601

[90] Wegner F 1994 Ann. Phys., NY 50677

Wegner F 2006 J. Phys. A: Math. Gen. 398221

[91] Kehrein S 2006 The Flow Equation Approach to Many Particle Systems (New York: Springer)

[92] Verdeny A, Mielke A and Mintert F 2013 Phys. Rev. Lett. 111 175301

Roy A and Das A 2015 Phys. Rev. B 91 121106(R)

[93] Thomson S J, Magano D and Schiro M 2020 arXiv:2009.03186 unpublished

[94] Vogl M, Laurell P, Barr A D and Fiete G A 2019 Phys. Rev. X 9021037

[95] Laptyeva T V, Kozinov E A, Meyerov I B, Ivanchenko M V, Denisov S V and Hänggi P 2016 Comput. Phys. Commun. 20185

[96] White S R and Feiguin A E 2004 Phys. Rev. Lett. 93 076401

Vidal G 2003 Phys. Rev. Lett. 91147902

[97] Nandy S, Sen A and Sen D 2017 Phys. Rev. X 7031034

[98] Nandy S, Sen A and Sen D 2018 Phys. Rev. B 98245144

[99] Maity S, Bhattacharya U, Dutta A and Sen D 2019 Phys. Rev. B 99 020306(R)

Bhattacharya U, Maity S, Banik U and Dutta A 2018 Phys. Rev. B 97184308

Maity S, Bhattacharya U and Dutta A 2018 Phys. Rev. B 98 064305

[100] Ray S, Sinha S and Sen D 2019 Phys. Rev. E 100052129

[101] Dumitrescu P T, Vasseur R and Potter A C 2018 Phys. Rev. Lett. 120070602

[102] Giergiel K, Kuroś A and Sacha K 2019 Phys. Rev. B 99 220303(R)

[103] Else D V, Ho W W and Dumitrescu P T 2020 Phys. Rev. X 10 021032

[104] Mukherjee B, Sen A, Sen D and Sengupta K 2020 Phys. Rev. B 102014301

[105] Mori T, Zhao H, Mintert F, Knolle J and Moessner R 2021 Phys. Rev. Lett. 127050602

[106] Verdeny A, Puig J and Mintert F 2016 Z. Naturforsch 71897

[107] Kamenev A and Levchenko A 2009 Adv. Phys. 58197 Kamenev A 2001 arXiv:cond-mat/0109316 unpublished

[108] See, for example, chapter 3.3 of Breuer H P and Petruccione F 2006 The Theory of Open Quantum Systems (Oxford: Oxford University Press)

[109] Robertson A and Galitski V M 2009 Phys. Rev. A 80063609 
Mankowsky R et al 2014 Nature $\mathbf{5 1 6} 71$

[110] Torre E G D, Diehl S, Lukin M D, Sachdev S and Strack P 2013 Phys. Rev. A 87023831

Sieberer L M, Huber S D, Altman E and Diehl S 2013 Phys. Rev. Lett. 110195301

Altman E, Sieberer L M, Chen L, Diehl S and Toner J 2015 Phys. Rev. X 5011017

Maghrebi M F and Gorshkov A V 2016 Phys. Rev. B 93014307 [111] Ghosh R, Dupuis N, Sen A and Sengupta K 2020 Phys. Rev. B 101245130
[112] Tsuji N, Oka T and Aoki H 2008 Phys. Rev. B 78 235124

Frank R 2013 New J. Phys. 15123030

Lee W-R and Park K 2014 Phys. Rev. B 89205126

Murakami Y, Tsuji N, Eckstein M and Werner P 2017 Phys. Rev. B 96045125

Qin T and Hofstetter W 2017 Phys. Rev. B 96075134

Qin T, Schnell A, Sengstock K, Weitenberg C, Eckardt A and Hofstetter W 2018 Phys. Rev. A 98033601

Lubatsch A and Frank R 2019 Symmetry 111246 